Павлов Александр Владимирович, кандидат технических наук, ассистент, кафедра начертательной геометрии, инженерной и компьютерной графики, Национальный технический университет Украины “КПИ”, пр. Победы, 37, Киев, Украина, 03056

E-mail: Alexander_mk@ukr.net

Павлов Андрей Владимирович, кандидат технических наук, научный сотрудник, Отдел информационных технологий и индуктивного моделирования, Международный научно-учебный центр информационных технологий и систем НАН Украины и МОН Украины, пр. Глушкова, 40, Киев, Украина, 03680

E-mail: andriypavlove@gmail.com

Павлов Владимир Анатольевич, кандидат технических наук, доцент, кафедра биомедицинской кибернетики, Национальный Технический Университет “КПИ”, пр. Победы, 37, Киев, Украина, 03056

E-mail:VPavlo@bk.ru

УДК 681.3.016:519.17:541.66

DOI: 10.15587/2313-8416.2014.27967

\title{
ПРОГНОЗИРОВАНИЕ КРИТИЧЕСКИХ СВОЙСТВ ФРЕОНОВ С ИСПОЛЬЗОВАНИЕМ НОВЫХ ИНВАРИАНТОВ ВЗВЕШЕННЫХ ГРАФОВ
}

\author{
(C) Ю. А. Кругляк, И. В. Передунова
}

Предлагается новый подход в задачах «структура - свойство» с использованием инвариантов полностью взвешенных графов для количественного описания критических свойств фреонов. Сформулирован общий принции построения топологических инвариантов полностью взвешенньх графов и предложены два новых инварианта, примененных для расчета критических свойств фреонов метанового, этанового и пропанового рядов без привлечения экспериментальных данных $u$ закономерностей.

Ключевые слова: фреон, критические свойства, критическая температура, критическое давление, критический объем, молекулярные графы, инварианты графов, взвешенные графы, индекс паросочетаний.

It is proposed a new approach to the "structure - property" problems with usage the invariants of the fully weighted graphs for quantitative description of the critical properties of freon. A general principle of topological invariants construction of fully weighted graphs is formulated and propose two new invariants applied to calculate the critical properties of freon of methane, ethane and propane series without the involvement of experimental data and patterns.

Keywords: freon, critical properties, critical temperature, critical pressure, critical volume, molecular graphs, graph invariants, fully weighted graphs, matching index.

\section{1. Введение}

Проблема установления связи между структурой молекул и свойствами молекулярных веществ сложна и многопланова. Причиной тому является не только разнообразие свойств веществ и трудности их экспериментального исследования как и структуры и свойств составляющих их молекул, но и следующее обстоятельство. Основными методами исследования связи «структура - свойство» являются методы регрессионного и корреляционного анализа и распознавания образов. Эти методы оперируют с численными характеристиками структуры молекул. Естественные численные характеристики молекулярной структуры такие как длины связей, валентные и диэдрические углы и разнообразные квантовохимические расчетные свойства молекул часто с успехом используются в методах распознавания образов, но мало пригодны для регрессионного и корреляционного анализа. Для этих методов предпочтительны интегральные численные характеристики молекулярной структуры. В этом плане большой интерес вызывают топологические инварианты, позволяющие описать структуру молекулы одним числом [1-3].

Под инвариантом молекулярного графа подразумевают такую величину, которая принимает одно и то же численное значение при любой произвольной нумерации вершин графа. Инварианты молекулярных графов получили в литературе название топологических индексов (ТИ). Под молекулярным графом подразумевают такой граф, вершины которого находятся во взаимнооднозначном соответствии с атомами рассматриваемой молекулы, а ребра - с химическими связями. Применение ТИ в качестве численных интегральных характеристик структуры молекул для установления связи «структура свойство» имеет ряд преимуществ. Во-первых, топологическое описание молекул опирается на хорошо разработанную теорию графов. Во-вторых, ТИ вычисляются только на основе структурной формулы молекул. В-третьих, для таких расчетов не требуется больших вычислительных ресурсов. 
2. Анализ литературных данных по прогнозированию критических свойств фреонов

Критические свойства фреонов были выбраны для изучения по трем причинам:

1) для их расчета не существует достаточно надежных методов при том, что экспериментальное измерение критических свойств фреонов вызывает значительные трудности;

2) фреоны являются удобными модельными соединениями для отработки методики параметризации предлагаемого нами метода, поскольку молекулы фреонов обладают простой структурой и разнообразием атомов;

3) изменение критических свойств в ряду галогензамещенных алканов характеризуется рядом особенностей, которые не позволяют описать зависимость этих свойств от строения молекул аддитивными методами.

Рассмотрим достоинства и недостатки наиболее известных методов расчета критических свойств веществ.

Первый путь - это восстановление неизвестных свойств по большому массиву известных экспериментальных данных согласно некоторым соотношениям, подтвержденным экспериментально на других соединениях [4]. Такой подход дает хорошие результаты, но он мало доступен, поскольку требует обработки больших массивов экспериментальных данных, при этом даже такие громоздкие вычисления не гарантируют требуемой точности.

Второй, чаще всего применяемый прием, использование эмпирических и полуэмпирических формул [5-8]. Все известные эмпирические и полуэмпирические методы могут быть разбиты на два типа по способу их применения.

К первому типу относятся методы, которые после параметризации на обучающей выборке веществ не требуют при расчете никаких дополнительных сведений о веществе кроме структуры его молекул и найденных параметров. Ко второму типу относятся такие методы, которые в качестве коррелирующего фактора используют другие экспериментально измеряемые свойства рассматриваемых веществ, что резко ограничивает возможности таких методов и делает невозможным их применение для расчета свойств еще не синтезированных веществ, а если и синтезированных, но необходимые свойства которых еще не измерены экспериментально.

Для расчета $T_{c}, K$ предложены методы только второго типа. Все они основаны на использовании обобщенного правила Гульдберга: $T_{c}=T_{\kappa u n, н} / \Theta$, где $T_{\text {киn,н }}$ - нормальная температура кипения, а величина $\Theta$ определяется разными авторами по разному:

$$
\Theta=0.567+\sum \Delta_{T}-\left(\sum \Delta_{T}\right)^{2} \text { через аддитивные }
$$

инкременты по Лидерсену [6],

$$
\Theta=2-\exp [A-B \sqrt{\ln (M / \rho)}] \quad \text { через молеку- }
$$
лярную массу $M$, плотность жидкости $\rho_{\text {кип, }}$ при $T_{\text {кип,н }}$ и так называемый $\psi$-фактор по методу Сладкова-1

и $\Theta=2-\exp (0.45-0.065 \sqrt{3.296+\ln \Pi)}$ через парахор П по методу Сладкова-2 [8].

Для расчета $V_{c}$ и $P_{c}$ предложены методы расчета первого типа. Критический объем вычисляется по инкрементам в методах Лидерсена [6] и Воулеса [7], а в методе Сладкова - через молекулярную массу и плотность жидкости при $T_{\text {кип,н }}$ с учетом типа соединения [8]. Критическое давление вычисляется через молекулярную массу и инкременты в методе Лидерсена [6], а в методе Сладкова - через $T_{c}$, молекулярную массу и плотность жидкости при $T_{\text {кun,н }}$ [8]. Как будет видно дальше, наши расчеты свидетельствуют, что эти методы прогнозирования $V_{c}$ и $P_{c}$ характеризуются большой погрешностью.

Известны попытки использовать ТИ для вычисления критических свойств веществ. Для алканов предложены зависимости их критических свойств от ТИ молекулярной связуемости $\chi$ индексов различного вида [9]. Однако, их применение ограничено только классом углеводородов, описываемых молекулярными графами без учета атомов водорода. Кроме того, весьма сложный вид найденных зависимостей не позволяет сделать даже приблизительные предположения о характере возникновения обнаруженных корреляций.

Выбранный нами класс фреонов характеризуется разнообразием атомов. Для их топологического описания мы предлагаем использовать полностью взвешенные графы, взвешенные как по вершинам, так и по ребрам. Задача состояла в том, чтобы найти такие инварианты полностью взвешенных графов, чтобы каждое из критических свойств коррелировало не только монотонно, но и линейно только с одним инвариантом. Для взвешивания вершин и ребер в настоящем исследовании использовались только числа. Однако, ничто не мешает взвешивание вершин и ребер в молекулярных графах вести изощренее, например, с помощью параметрических функций, векторов, операторов и еще более сложных математических объектов.

\section{3. Проблема выбора инвариантов и параметров \\ Требование монотонной зависимости} свойства от ТИ является достаточно жестким условием, позволяющем выделить на первом этапе исследования ограниченный круг претендентов среди множества ТИ, а также найти границы возможного изменения весов вершин и ребер. Достигается это путем решения ряда неравенств типа $I\left(G_{i}\right)>I\left(G_{j}\right)$, если $F\left(S_{i}\right)>F\left(S_{j}\right)$, где $F\left(S_{i}\right)-$ 
значение физического свойства соединения $S_{i}$, а $I\left(G_{i}\right)$ - инвариант соответствующего молекулярного графа.

Параметризацию целесообразно начинать с простейших соединений из рассматриваемого класса веществ. В нашем случае это галогенпроизводные метана. Оказывается возможным подобные неравенства решить аналитически. При этом выясняются сразу два вопроса:

1) возможно ли вообще удовлетворение этих неравенств при каких-либо значениях параметров (весов вершин и ребер), тем самым решается вопрос о принципиальной применимости того или иного ТИ;

2) если это возможно, то в каких границах должны изменяться параметры.

Начинать анализ удобно с выделения подрядов выбранного класса соединений, образованных последовательным замещением одного параметризуемого атома на другой. В этом случае неравенства оказываются достаточно простыми, а кроме того мы избегаем опасности появления ошибки из-за неточности экспериментальных данных, поскольку такие соединения, как правило, различаются по свойствам на значительную величину. Если же исследовать соединения в порядке возрастания или убывания рассматриваемого свойства, то противоречия в справочных данных часто не позволяют определить даже порядок следования веществ.

Рассмотрим для примера $T_{c}$. При замещении атомов водорода метана атомами фтора $T_{c}$ увеличивается в следующем порядке (литературные источники используемых экспериментальных данных будут приведены позже):

$T_{c}\left(\mathrm{CH}_{4}\right)=190.6, \quad T_{c}\left(\mathrm{CF}_{4}\right)=227.5, \quad T_{c}\left(\mathrm{CHF}_{3}\right)=229.0$,

$T_{c}\left(\mathrm{CH}_{3} \mathrm{~F}\right)=317.7, \quad T_{c}\left(\mathrm{CH}_{2} \mathrm{~F}_{2}\right)=351.6 \mathrm{~K}$,

в то время как для хлорзамещенных метана $T_{c}$ увеличивается последовательно при переходе от $\mathrm{CH}_{4}$ к $\mathrm{CCl}_{4}$.

Рассмотрим ТИ класса ${ }^{2} \chi$ модифицированный нами для взвешенных графов в виде [10]

$$
{ }^{2} \tilde{\chi}=\sum_{i, j, k-a d j} a_{i} a_{j} a_{k} b_{i j} b_{j k} .
$$

Пусть в ряду фторзамещенных метана $a_{\mathrm{H}}, a_{\mathrm{C}}, a_{\mathrm{F}}$ есть веса соответствующих вершин, а $b_{\mathrm{H}} \equiv b_{\mathrm{CH}}, b_{\mathrm{F}} \equiv b_{\mathrm{CF}}-$ веса ребер. Тогда

$$
\begin{aligned}
{ }^{2} \tilde{\chi}\left(\mathrm{CH}_{4}\right) & =6 a_{\mathrm{C}} a_{\mathrm{H}}^{2} b_{\mathrm{H}}^{2}, \quad{ }^{2} \tilde{\chi}\left(\mathrm{CF}_{4}\right)=6 a_{\mathrm{C}} a_{\mathrm{F}}^{2} b_{\mathrm{F}}^{2}, \\
{ }^{2} \tilde{\chi}\left(\mathrm{CHF}_{3}\right) & =3 a_{\mathrm{C}} a_{\mathrm{H}} a_{\mathrm{F}} b_{\mathrm{H}} b_{\mathrm{F}}+3 a_{\mathrm{C}} a_{\mathrm{F}}^{2} b_{\mathrm{F}}^{2}, \\
{ }^{2} \tilde{\chi}\left(\mathrm{CH}_{3} \mathrm{~F}\right) & =3 a_{\mathrm{C}} a_{\mathrm{H}} a_{\mathrm{F}} b_{\mathrm{H}} b_{\mathrm{F}}+3 a_{\mathrm{C}} a_{\mathrm{H}}^{2} b_{\mathrm{H}}^{2}, \\
{ }^{2} \tilde{\chi}\left(\mathrm{CH}_{2} \mathrm{~F}_{2}\right) & =a_{\mathrm{C}} a_{\mathrm{H}}^{2} b_{\mathrm{H}}^{2}+a_{\mathrm{C}} a_{\mathrm{F}}^{2} b_{\mathrm{F}}^{2}+4 a_{\mathrm{C}} a_{\mathrm{H}} a_{\mathrm{F}} b_{\mathrm{H}} b_{\mathrm{F}} .
\end{aligned}
$$

Разделим эти выражения на $a_{\mathrm{C}} a_{\mathrm{H}}^{2} b_{\mathrm{H}}^{2}$, что не влияет на порядок следования значений индекса ${ }^{2} \tilde{\chi}$ по возрастанию. Примем $a \equiv a_{F} / a_{H}, b \equiv b_{F} / b_{H}$, тогда должны выполняться неравенства:
$6<6 a^{2} b^{2}<3 a b(1+a b)<3(1+a b)<1+a^{2} b^{2}+4 a b$. (3)

Из первого неравенства следует, что $|a b|>1$. Пусть $a b>1$, тогда $1+a b>2$ и из третьего неравенства получаем $a b<1$, что противоречит исходной посылке. Допустим, что $a b<-1$, тогда $1+a b<0$ и из третьего неравенства при сокращении на $1+a b$ знак неравенства меняется на противоположный и получаем $a b>1$, что опять противоречит исходной посылке. Следовательно, ни при каких значениях $a$ и $b$ невозможно удовлетворить рассматриваемую последовательность неравенств (3), и как следствие этого индекс ${ }^{2} \tilde{\chi}$ не может описать изменение $T_{c}$ при замещении атомов водорода в метане атомами фтора.

Аналогичным образом были проверены все многочисленные известные нам ТИ, и ни один из них не попал в число претендентов для прогнозирования $T_{c}$ и $P_{c}$. Покажем, что на эту роль может претендовать предлагаемый нами новый ТИ индекс паросочетаний.

\section{4. Обоснование индекса паросочетаний}

В корреляциях «структура - свойство» часто используется полином паросочетаний (ПП) [1114]. Он определяется следующим образом:

$$
\alpha(G, x)=\sum_{k=0}^{[p / 2]}(-1)^{k} P(G, k) x^{p-2 k},
$$

где $p$ - число вершин графа $G ; P(G, k)$ - число $k$ паросочетаний; $P(G, 0)=1$. При этом $k$ паросочетание трактуется как $k$ попарно не связанных ребер. Аналогично определяется многочлен Хозоя [15]:

$$
Q(G, x)=\sum_{k=0}^{[p / 2]} P(G, k) x^{k} .
$$

Есть в литературе и другое толкование самого понятия паросочетания и, как следствие, соответствующего полинома. В [16] дается дословно следующее определение: «Под паросочетанием $M$ графа $G$ мы будем понимать основной подграф графа $G$, состоящий только из ребер и вершин. Если $M$ содержит $k$ ребер, то $M$ называется $k$ паросочетанием. Ясно, что если $G$ содержит $p$ вершин, то $k$-паросочетание в $G$ содержит $p-2 k$ вершин». И далее следует формула для ПП:

$$
M(G, w)=\sum_{k=0}^{[p / 2]} a_{k} w_{1}^{p-2 k} w_{2}^{k},
$$

где $w=\left(w_{1}, w_{2}\right)$ - весовой 2-вектор, компоненты которого образуют полином; $w_{1}-$ вес вершин; $w_{2}-$ вес ребер; $a_{k}$ - число $k$-паросочетаний.

Понятно, что число $a_{k}=P(G, k)$, а полином $\alpha(G, x)$ является частным случаем $M(G, w)$ при $w_{2}=-1$, т. e.

$$
\alpha(G, x)=M(G,(x,-1))
$$


Приведенное определение интересно тем, что открывает большие возможности для описания гетероатомных молекул. Полином $\alpha(G, x)$ первоначально использовался лишь для характеристики углеводородов, т. е. таких соединений, при описании которых в виде графа без учета атомов водорода нет необходимости придавать веса вершинам и ребрам. Именно поэтому таким эффективным для этого класса веществ оказался полином $Q(G, x)$ и его выражение при $x=1$ (так называемый индекс Хозоя $[15,17])$.

Переход от углеводородов к гетероатомным молекулам - задача довольно сложная. При использования ТИ для описания некоторого свойства гетероатомной молекулы необходимо ввести веса вершин и ребер. Они должны отражать влияние атомов и связей на величину исследуемого свойства вещества. Применительно к гетеросопряженным системам такой переход для характеристического полинома совершается естественным образом в соответствии с методом Хюккеля, вопрос лишь в адекватном подборе значений кулоновских и резонансных интегралов. Попытки преобразовать ПП применительно к гетероатомным молекулам нам не известны. Для гетеросопряженных молекул вводятся веса вершин и ребер в рекуррентной форме [18]:

$$
\begin{gathered}
\alpha\left(G_{h}, x\right)=\alpha(G, x)-h \alpha(G-v, x), \\
\alpha\left(G_{k}, x\right)=\alpha(G-l, x)-k^{2} \alpha(G-(l), x),
\end{gathered}
$$

где $h$ - вес петли при вершине, соответствующей гетероатому $v ; k$ - вес ребра $l ; G-v-$ граф с удаленной вершиной $v ; G-l-$ удаление ребра $l$; $G-(l)$ - удаление ребра $l$ с инцидентными ему ребрами. Такая форма обосновывается аналогией с характеристическими полиномами. Однако, она применима лишь для класса гетеросопряженных молекул и, несмотря на свою наглядность, реализуется алгоритмами со сложной логической структурой.

Уместно вспомнить о представлении ПП в форме $M(G, w)$. Если в формуле (6) считать, что веса ребер $w_{2}$ не равны между собой, а характеризуют химическую связь, соответствующую данному ребру, то для каждого паросочетания $M_{k}^{j}$ вместо $w_{2}^{k}$ можно записать $\prod_{i=1}^{k} b_{i}^{j}$, где $b_{i}^{j}-$ вес $i$-го ребра, принадлежащего $j$-му $k$-паросочетанию $M_{k}^{j}$. Аналогично можно раскрыть $w_{1}^{p-2 k}$ в (6). Если считать различными веса вершин, то $w_{1}^{p-2 k}$ преобразуется в $\prod_{i=1}^{p-2 k} a_{i}^{j}$, где $a_{i}^{j}$ принадлежат $M_{k}^{j}$. В результате каждому $k$-паросочетанию соответствует произведение весов входящих в него вершин и ребер, и число таких произведений равно $P(G, k)$. Просуммировав, получаем вместо $P(G, k) w_{1}^{p-2 k} w_{2}^{k}$ число

$$
\tilde{P}(G, k)=\sum_{j=1}^{P(G, k)}\left(\prod_{i=1}^{p-2 k} a_{i}^{j}\right)\left(\prod_{i=1}^{k} b_{i}^{j}\right) .
$$

Отсюда легко получается предлагаемый нами индекс паросочетаний (ИП)

$$
R(G)=\sum_{k=1}^{[p / 2]} \tilde{P}(G, k) .
$$

Числа $\tilde{P}(G, k)$ можно назвать значениями $k$ гетеропаросочетаний и использовать их в соответствующих полиномах вместо $P(G, k)$. В случае, если веса вершин и ребер равны единице, то $\tilde{P}(G, k)=P(G, k)$, и предлагаемый нами индекс паросочетаний превращается в индекс Хозоя. Отличие предлагаемого нами метода построения ИП от использовавшихся подходов ранее заключается в том, что при суммировании по подграфам в каждом слагаемом участвуют как характеристики элементов этих подграфов (в нашем случае ребер), так и характеристики непокрываемых этими подграфами вершин, что позволяет в каждом слагаемом учесть влияние окружения.

\section{5. Вычисление индекса паросочетаний}

Для нахождения чисел $P(G, k)$ предложены эффективные алгоритмы, однако, задача вычисления $\tilde{P}(G, k)$ предполагает перебор всех паросочетаний. Представляется целесообразным свести этот процесс к перебору полных подграфов с последовательным увеличением их порядка.

Пусть $G$ взвешенный граф с $p$ вершинами и $q$ ребрами, $A$ - вектор размерности $p$ весов вершин, $B$ - вектор размерности $q$ весов ребер. Граф $G$ может задаваться либо бинарной матрицей смежности, либо перечислением пар смежных вершин. В обоих случаях не составляет труда построить матрицу смежности $M$ реберного графа $L(G)$.

Вершинами графа $L(G)$ являются ребра графа $G$. Две вершины графа $L(G)$ смежны тогда и только тогда, когда смежны соответствующие им ребра графа $G[19]$. Поскольку матрица $M$ симметричная, то, сохраняя всю информацию о графе, можно считать ее верхней треугольной

$$
M=\left\{\delta_{i j}\right\}, \quad i, j=1,2, \ldots, q
$$

$$
\delta_{i j}= \begin{cases}1, & \text { если } j>i \text { и вершины графа } L(G) \text { смежные, } \\ 0 & \text { в противном случае. }\end{cases}
$$

Рассматривая $\delta_{i j}$ как битовые константы, получаем матрицу

$$
\bar{M}=\left\{\delta_{i j}^{\prime}\right\}
$$

где

$$
\delta_{i j}^{\prime}= \begin{cases}\bar{\delta}_{i j}, & \text { если } j>i, \\ 0, & \text { если } j \leq i .\end{cases}
$$

Это матрица смежности дополнения графа $L(G)$. Если $\overline{L(G)}$ есть дополнение графа $L(G)$, то 
любой полный подграф $\overline{L(G)}$ с $k$ вершинами соответствует набору ребер $k$-паросочетания графа $G$.

Bce $k$-паросочетания можно объединить в матрицу

$$
W_{k}=\left(\begin{array}{cccc}
l_{11} & l_{12} & \cdots & l_{1 L} \\
l_{21} & l_{22} & \cdots & l_{2 L} \\
\cdots & \cdots & \cdots & \cdots \\
l_{k 1} & l_{k 2} & \cdots & l_{k L}
\end{array}\right)
$$

где $L=P(G, k), j$-ый столбец представляет собой номера ребер, входящих в $j$-ое $k$-паросочетание графа $G$ или номера вершин полного подграфа графа $\overline{L(G)}$. Таким образом, несложными преобразованиями, легко осуществимыми алгоритмически, задача сводится к перечислению всех полных подграфов графа $\overline{L(G)}$.

Это перечисление проводится простым и эффективным способом. При $k=1$ матрица $W_{1}$ представляет собой строку номеров вершин: $W_{1}=(1,2, \ldots, q)$, а при $k=2$ матрица $W_{2}-$ это индексы единиц в матрице $M$, или номера пар смежных вершин.

Пусть найдена матрица $W_{k}$, тогда для вычисления матрицы $W_{k+1}$ с каждым ј-ым столбцом матрицы $W_{k}$ производится следующая операция. Строки матрицы $M$ рассматриваются как логические векторы $X_{n}$. Ищется вектор

$$
X=\left(X_{1}, X_{2}, \ldots, X_{n}\right), \quad n \in\left\{l_{k j}\right\},
$$

где $\left\{l_{k j}\right\}$ - номера вершин, перечисленных в $j$-ом столбце матрицы $W_{k}$. Когда $X=0$, никаких действий не производится, в противном случае, если $\left\{l_{k+1, f}\right\} \quad(1<f<q)-$ номера единиц в векторе $X$, то в матрицу $W_{k+1}$ заносятся столбцы $\left(l_{1 j}, l_{2 j}, \ldots l_{k j}, l_{k+1, f}\right)^{T}$. Процедура продолжается до тех пор, пока для каждого столбца из $W_{k}$ вектор $X$ не будет равен нулю.

В качестве примера рассмотрим вычисления ИП для некоторого галогенпроизводного пропана:

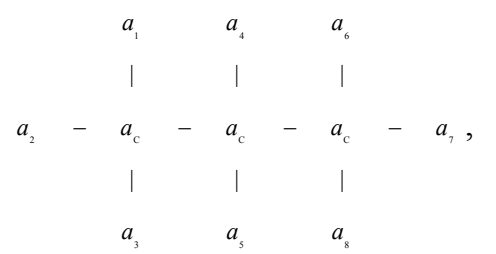

где вершины обозначены весами. Связи этого графа задаются таблицей:

\begin{tabular}{|l|l|l|l|l|l|l|l|l|l|l|}
\hline $\begin{array}{l}\text { Номер } \\
\text { ребра }\end{array}$ & 1 & 2 & 3 & 4 & 5 & 6 & 7 & 8 & 9 & 10 \\
\hline $\begin{array}{l}\text { Инцидентные } \\
\text { вершины }\end{array}$ & 9 & 9 & 9 & 10 & 10 & 11 & 11 & 11 & 9 & 10 \\
\hline Вес ребра & $b_{1}$ & $b_{2}$ & $b_{3}$ & $b_{4}$ & $b_{5}$ & $b_{6}$ & $b_{7}$ & $b_{8}$ & $b_{\mathrm{C}}$ & $b_{\mathrm{C}}$ \\
\hline
\end{tabular}

Составляем матрицы:

$$
M=\left(\begin{array}{l}
0110000010 \\
0010000010 \\
0000000010 \\
0000100011 \\
0000000011 \\
0000001101 \\
0000000101 \\
0000000001 \\
0000000001 \\
0000000000
\end{array}\right), \quad \bar{M}=\left(\begin{array}{l}
0001111101 \\
0001111101 \\
0001111101 \\
0000011100 \\
0000011100 \\
0000000010 \\
0000000010 \\
0000000010 \\
0000000000 \\
0000000000
\end{array}\right) .
$$

Ищем полные подграфы:

$W_{1}=(12345678910)$,

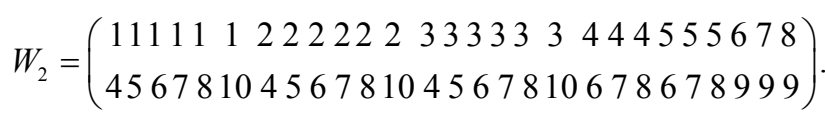

Просматриваем последовательно столбцы $W_{2}$. Для первого столбца

$$
X=X_{1} \wedge X_{4}=(0000011100),
$$

следовательно, первые три столбца $W_{3}$ будут выглядеть таким образом: $\quad \begin{array}{lllll}1 & 1 & 1 \\ & 4 & 4 & 4 \text {.Остальные }\end{array}$ столбцы получаем аналогично:

$$
W_{3}=\left(\begin{array}{l}
111111222222333333 \\
444555444555444555 \\
678678678678678678
\end{array}\right) .
$$

Любая конъюнкция для $W_{3}$ равна нулю. Следовательно, максимальным будет 3паросочетание. Для него, например, на основе матрицы $W_{3}$ можно записать:

$\tilde{P}(G, 3)=b_{1} b_{4} b_{6} a_{2} a_{3} a_{5} a_{7} a_{8}+b_{1} b_{4} b_{8} a_{2} a_{3} a_{5} a_{6} a_{7}+\ldots+b_{3} b_{5} b_{8} a_{1} a_{2} a_{4} a_{6} a_{7}$.

При программной реализации алгоритма многие действия можно упростить и объединить.

6. Общая схема подбора параметров и прогнозирования свойств веществ

Предлагаемая нами техника прогнозирования физико-химических свойств относится прежде всего к веществам, свойства которых определяются топологией, составом и свойствами молекул, образующих вещество. С незначительными модификациями она может быть также использована для более широкого круга веществ, когда необходимо учесть влияние ближайшего окружения и среды. Представим схему прогнозирования в виде этапов, а затем продемонстрируем ее на примере фреонов.

Этап 1. Выявление ряда веществ, для которых возможность применения ТИ и ограничения на параметры можно определить аналитически. 
Рассмотрение подрядов, решение соответствующих неравенств, выделение существенных ограничений на значения весов вершин и ребер.

Этап 2. Оптимизация параметров в выделенном ряду. Здесь надо учитывать несколько моментов. Во-первых, оптимизация может проводиться как с использованием методов нулевого порядка (например, симплекс-методов), так и прямым перебором параметров с разным шагом, если найденные ограничения на параметры позволяют это сделать за реальное время. Вовторых, как при обычной оптимизации, так и при переборе следует учитывать возможную разницу в чувствительности ТИ к значениям весов вершин и ребер, от этого зависит и точность, с которой надо подбирать параметры. В-третьих, учитывая разную природу параметров, характеризующих графы, и параметров зависимости физико-химического свойства от инварианта, их надо оптимизировать разными методами. Если, например, за критерий качества аппроксимации принято среднеквадратичное отклонение, то для текущих параметров графа вычисляются инварианты, которые считаются значениями независимой переменной для метода наименьших квадратов, этим методом определяются коэффициенты зависимости физико-химической величины от значений ТИ, а найденные среднеквадратичные отклонения являются значениями критериальной функции для оптимизации параметров графа. В-четвертых, вся выборка веществ с известными физикохимическими свойствами должна быть разбита на обучающую, на которой проводится оптимизация, и контрольную выборку для проверки прогнозирующей способности модели. Это требование остается в силе для всех последующих этапов.

Этап 3. Расширение класса рассматриваемых веществ с добавлением нескольких новых параметров, а определенные ранее параметры считаются фиксированными. Здесь может появиться возможность оптимизировать параметры более простым и эффективным методом. В том случае, если при выражении инварианта в общем виде через известные и неизвестные параметры последние входят в виде суммы с некоторыми коэффициентами, то можно считать числовые значения неизвестных параметров независимыми переменными для линейной регрессионной модели и стандартными методами множественной линейной регрессии найти искомые параметры.

Этап 4. Дальнейшее усложнение структуры рассматриваемых веществ. Если появятся новые параметры, то повторить этап 3. В противном случае по опорным веществам найти коэффициенты регрессионного уравнения и проверить прогнозирующую способность.

Этап 5. Генерация веществ, физикохимические свойства которых можно рассчитать с помощью найденных параметров, и расчет этих свойств.

\section{7. Параметризация и расчет $\boldsymbol{T}_{c}$ и $\boldsymbol{P}_{c}$ фреонов}

Прогнозирование $T_{c}$ и $P_{c}$ фреонов выполнено с использованием предложенного нами ИП (10). Никакие известные нам ТИ, модифицированные для взвешенных графов, не выдержали проверки уже на первом этапе. Это, однако, не исключает возможности генерации другого ТИ, пригодного для стоящей перед нами задачи.

Этап 1. Рассматриваем галогенопроизводные метана, выделяем подряд

$$
\begin{array}{lllll}
\mathrm{C} X_{4} & \mathrm{C} X_{3} Y & \mathrm{C}_{2} Y_{2} & \mathrm{C} X Y_{3} & \mathrm{C} Y_{4}
\end{array}
$$

и найдем ограничения на параметры вершин и ребер при увеличении изучаемого свойства в той же последовательности. Выпишем последовательно в соответствии с (16) в общем виде выражения для ИП:

$4 b_{X} a_{X}^{3}, 3 b_{X} a_{X}^{2} a_{Y}+b_{Y} a_{X}^{3}, 2 b_{X} a_{X} a_{Y}^{2}+2 b_{Y} a_{X}^{2} a_{Y}, b_{X} a_{Y}^{3}+3 b_{Y} a_{X} a_{Y}^{2}, 4 b_{Y} a_{Y}^{3} .(17)$

Разделим все выражения на $b_{X} a_{X}^{3}$ и внесем это сомножитель в дальнейшем в коэффициент при ИП. Положим $a=a_{Y} / a_{X}, b=b_{Y} / b_{X}$ и получим следующую последовательность неравенств:

$$
4<3 a+b<2 a(a+b)<3 b a^{2}+a^{3}<4 b a^{3} .
$$

Найдем ограничения на параметр $b$ сразу из всех четырех неравенств:

$1: 4<3 a+b \Rightarrow b>4-3 a$,

$2: 3 a+b<2 a(a+b) \Rightarrow(2 a-1) b>3 a-2 a^{2}$,

a) если $2 a-1>0$, то $b>\frac{3 a-2 a^{2}}{2 a-1}$,

б) если $2 a-1<0$, то $b<\frac{3 a-2 a^{2}}{2 a-1}$,

$3: 2 a(a+b)<3 b a^{2} \Rightarrow b>\frac{a-a^{2}}{2}$,

$4: 3 b a^{2}+a^{3}<4 b a^{3} \Rightarrow b(4 a-3)>a$,

a) если $4 a-3>0$, то $b>\frac{a}{4 a-3}$,

б) если $4 a-3<0$, то $b<\frac{a}{4 a-3}$.

Выясним какое из первых двух неравенств более строгое. Для этого рассмотрим разность:

$$
\frac{3 a-2 a^{2}}{2 a-1}-(4-3 a)=\frac{4(a-1)^{2}}{2 a-1} \text {. }
$$

Начнем со случая (2a), тогда из (27) следует

$$
\frac{3 a-2 a^{2}}{2 a-1}>4-3 a,
$$

так что в дальнейшем надо проверять условие (21). Перейдем к случаю (2б), тогда

$$
4-3 a<b<\frac{3 a-2 a^{2}}{2 a-1} \text {. }
$$

Из (27) следует, что 


$$
4-3 a>\frac{3 a-2 a^{2}}{2 a-1}
$$

и мы приходим к противоречию с (29), значит $a>0.5$. Из (23) $b>\frac{a-a^{2}}{2}$, сравниваем с

Предположим, что (21) более строгое неравенство, тогда

$\frac{3 a-2 a^{2}}{2 a-1}>\frac{a-a^{2}}{2} \Rightarrow 6-4 a>(2 a-1)(1-a) \Rightarrow 2 a^{2}-7 a+7>0$,

т. е. предположение верно.

Для сравнения условий (25) и (26) с (21) рассмотрим разность

$$
\frac{3 a-2 a^{2}}{2 a-1}-\frac{a}{4 a-3}=\frac{-8 a(a-1)^{2}}{(2 a-1)(4 a-3)}
$$

В случае $4 \mathrm{a}$ из (31) следует, что $\frac{a}{4 a-3}>\frac{3 a-2 a^{2}}{2 a-1}$, так что неравенство (25) более строгое. Случай 46 не возможен, т.к. из (31) следует, что при $4 a-3$ имеет место неравенство $\frac{a}{4 a-3}<\frac{3 a-2 a^{2}}{2 a-1}$. Отсюда получаем окончательные ограничения на параметры:

$$
a>0.75, \quad b>\frac{a}{4 a-3} .
$$

Такая последовательность возрастания свойства характерна для $T_{c}$ при:

1) $X=\mathrm{H}, Y=\mathrm{Cl}$; 2) $X=\mathrm{H}, Y=\mathrm{Br}$;

3) $X=\mathrm{F}, Y=\mathrm{Cl}$; 4) $X=\mathrm{F}, Y=\mathrm{Br}$; 5) $X=\mathrm{Cl}, Y=\mathrm{Br}$,

а для $P_{c}$ при:

1) $X=\mathrm{F}, Y=\mathrm{Cl}$; 2) $X=\mathrm{F}, Y=\mathrm{Br} ; 3) X=\mathrm{Cl}, Y=\mathrm{Br}$.

Последовательность, отличная от рассмотренной, обнаруживается для $T_{c}$ при $X=\mathrm{H}, Y=\mathrm{F}$. В этом подряду $T_{c}$ возрастает в следующем порядке:

$$
\mathrm{CH}_{4}, \mathrm{CF}_{4}, \mathrm{CHF}_{3}, \mathrm{CH}_{3} \mathrm{~F}, \mathrm{CH}_{2} \mathrm{~F}_{2} .
$$

Соответствующие неравенства могут быть удовлетворены при следующих ограничениях на параметры:

$$
\begin{aligned}
& \text { 1) } 0.5<a<\sqrt{3} / 3, \quad b>\frac{3 a-2 a^{2}}{2 a-1}, \\
& \text { 2) } 0.75<a<1, \quad \frac{1}{a^{3}}<b<\frac{3 a-a^{3}}{3 a^{2}-1} .
\end{aligned}
$$

Для $P_{c} \quad$ при $\quad X=\mathrm{H}, Y=\mathrm{F} \quad$ и $\quad X=\mathrm{H}, Y=\mathrm{Cl}$ вещества в порядке возрастания свойства располагаются следующим образом:

$$
\mathrm{CY}_{4}, \quad \mathrm{C} X_{4}, \quad \mathrm{C} X Y_{3}, \quad \mathrm{C} X_{2} Y_{2}, \quad \mathrm{C} X_{3} Y .
$$

Это приводит к следующим ограничениям на параметры:

$$
\begin{aligned}
& \text { 1) } 0.657<a<1, \frac{4-a^{3}}{3 a^{2}}<b<\frac{3 a-2 a^{2}}{2 a-1} \text {, } \\
& \text { 2) } 0.5<a<0.657, \frac{4-a^{3}}{3 a^{2}}<b<\frac{1}{a^{3}} \text {. }
\end{aligned}
$$

При $X=\mathrm{H}, Y=\mathrm{Br}$ последовательности

$$
\mathrm{C} X_{4}, \quad \mathrm{C} Y_{4}, \quad \mathrm{C} X Y_{3}, \quad \mathrm{C} X_{2} Y_{2}, \quad \mathrm{C} X_{3} Y
$$

соответствуют ограничения:

$$
\begin{aligned}
& \text { 1) } 0<a<0.5, \quad b>a^{-3} \text {, } \\
& \text { 2) } 0.5<a<0.657, \frac{1}{a^{3}}<b<\frac{3 a-2 a^{2}}{2 a-1} \text {. }
\end{aligned}
$$

В процессе анализа других подрядов по мере расширения числа веществ были найдены еще более строгие неравенства и иные соотношения между параметрами. Здесь же мы демонстрируем анализ неравенств на первом этапе.

Этап 2. Этот этап осуществлялся с помощью разработанного нами комплекса программ [], позволяющего проводить оптимизацию параметров как методом симплекса, так и перебором параметров в заданных границах с выбранным шагом для каждого параметра. Была учтена возможность сокращения перебора путем введения соотношений между параметрами. В качестве критериальной функции для оптимизации весов вершин и ребер использовалась подпрограмма, реализующая линейный метод наименьших квадратов, но была возможность использовать и другие методы.

При поиске начального приближения для последующей оптимизации проводился перебор параметров вершин с шагом 0.001 и параметров ребер с шагом 0.01 с учетом различия в чувствительности ИП к их изменениям. Результаты расчетов представлены в табл. 1, 2 . 
Таблица 1

Экспериментальные и расчетные значения $T_{c}, K$ фреонов метанового ряда

\begin{tabular}{|c|c|c|c|c|c|c|c|c|c|c|c|}
\hline \multirow[t]{2}{*}{ №̄ ${ }^{1}$} & \multirow[t]{2}{*}{ Ключ $^{2}$} & \multicolumn{2}{|c|}{$\begin{array}{l}\text { Эксперимент. } \\
\text { данные }^{3}\end{array}$} & \multicolumn{2}{|c|}{$\begin{array}{c}\text { Метод } \\
\text { Сладкова-1 }\end{array}$} & \multicolumn{2}{|c|}{$\begin{array}{c}\text { Метод } \\
\text { Сладкова-2 }\end{array}$} & \multicolumn{2}{|c|}{$\begin{array}{c}\text { Метод } \\
\text { Лидерсена }\end{array}$} & \multicolumn{2}{|c|}{ Наш расчет ${ }^{5}$} \\
\hline & & Min & Max & $T_{c}$ & $E, \%$ & $T_{c}$ & $E, \%$ & $T_{c}$ & $E, \%$ & $T_{c}$ & $E \%$ \\
\hline $01^{\#}$ & 0000 & 190.55 & 191.60 & 181.32 & 4.84 & 181.24 & 4.89 & 189.92 & 0.33 & 196.70 & 2.67 \\
\hline $02^{\#}$ & 0001 & 317.70 & 317.80 & 310.81 & 2.17 & 313.50 & 1.32 & 320.37 & 0.81 & 319.48 & 0.53 \\
\hline $03^{\#}$ & 0002 & 416.20 & 416.30 & 396.25 & 4.79 & 399.80 & 3.94 & 413.42 & 0.67 & 400.46 & 3.78 \\
\hline 04 & 0003 & 462.16 & 467.00 & 437.91 & 5.24 & 442.06 & 4.35 & 464.02 & 0.00 & 438.63 & 5.09 \\
\hline $05^{\#}$ & 0011 & 351.55 & 351.60 & 354.15 & 0.73 & 357.77 & 1.75 & 357.06 & 1.55 & 340.14 & 3.25 \\
\hline $06^{*}$ & 0012 & 424.83 & 424.83 & 417.88 & 1.64 & 422.56 & 0.53 & 426.35 & 0.36 & 421.35 & 0.82 \\
\hline $07 *$ & 0013 & 468.14 & 468.14 & 456.49 & 2.49 & 462.01 & 1.31 & 472.73 & 0.98 & 464.39 & 0.82 \\
\hline $08^{\#}$ & 0022 & 509.85 & 510.00 & 492.37 & 3.43 & 497.74 & 2.37 & 506.57 & 0.64 & 510.74 & 0.14 \\
\hline $09 *$ & 0023 & 555.49 & 555.49 & 532.99 & 4.05 & 540.55 & 2.69 & 557.48 & 0.36 & 558.19 & 0.49 \\
\hline 10 & 0033 & 583.00 & 605.70 & 577.74 & 0.90 & 584.73 & 0.00 & 611.08 & 0.92 & 607.80 & 0.36 \\
\hline $11^{\#}$ & 0111 & 298.89 & 299.45 & 304.14 & 1.57 & 307.66 & 2.75 & 303.61 & 1.39 & 301.91 & 0.82 \\
\hline $12^{\#}$ & 0112 & 369.13 & 369.30 & 365.93 & 0.87 & 371.02 & 0.47 & 369.86 & 0.15 & 378.23 & 2.42 \\
\hline $13^{*}$ & 0113 & 411.11 & 411.11 & 409.88 & 0.30 & 409.68 & 0.35 & 413.91 & 0.68 & 421.34 & 2.49 \\
\hline $14^{\#}$ & 0122 & 451.50 & 451.65 & 447.31 & 0.93 & 447.15 & 0.96 & 449.40 & 0.47 & 462.12 & 2.32 \\
\hline $15^{*}$ & 0123 & 507.54 & 507.54 & 501.27 & 1.23 & 500.80 & 1.33 & 509.77 & 0.44 & 509.57 & 0.42 \\
\hline $16^{*}$ & 0133 & 543.53 & 543.53 & 534.05 & 1.74 & 533.36 & 1.87 & 549.88 & 1.17 & 559.36 & 2.91 \\
\hline $17^{\#}$ & 0222 & 536.40 & 536.60 & 528.93 & 1.39 & 527.52 & 1.66 & 533.84 & 0.48 & 554.36 & 3.31 \\
\hline $18^{*}$ & 0223 & 585.43 & 585.43 & 572.51 & 2.21 & 571.77 & 2.33 & 585.77 & 0.06 & 606.57 & 3.61 \\
\hline 19 & 0233 & 630.00 & 654.41 & 636.87 & 0.00 & 635.88 & 0.00 & 659.57 & 0.82 & 661.37 & 1.10 \\
\hline 20 & 0333 & 656.00 & 684.94 & 663.14 & 0.00 & 662.02 & 0.00 & 695.30 & 1.58 & 718.87 & 5.17 \\
\hline $21^{\#}$ & 1111 & 227.50 & 227.60 & 232.79 & 2.28 & 233.12 & 2.42 & 228.74 & 0.50 & 232.04 & 2.30 \\
\hline $22^{\#}$ & 1112 & 301.75 & 302.00 & 304.80 & 0.93 & 305.28 & 1.09 & 302.41 & 0.13 & 301.66 & 0.03 \\
\hline $23^{\#}$ & 1113 & 339.90 & 340.70 & 341.85 & 0.34 & 342.11 & 0.42 & 343.32 & 0.77 & 342.14 & 0.42 \\
\hline $24^{\#}$ & 1122 & 385.00 & 385.20 & 385.16 & 0.00 & 385.50 & 0.08 & 384.84 & 0.04 & 377.26 & 2.01 \\
\hline $25^{\#}$ & 1123 & 426.73 & 427.80 & 425.10 & 0.38 & 425.40 & 0.31 & 429.96 & 0.51 & 421.77 & 1.16 \\
\hline 26 & 1133 & 470.00 & 472.08 & 468.97 & 0.22 & 469.60 & 0.21 & 479.94 & 1.67 & 468.57 & 0.30 \\
\hline $27^{\#}$ & 1222 & 471.00 & 471.50 & 467.61 & 0.72 & 467.66 & 0.71 & 469.95 & 0.22 & 460.32 & 2.27 \\
\hline $28^{*}$ & 1223 & 516.26 & 516.26 & 511.20 & 0.98 & 510.98 & 1.02 & 519.68 & 0.66 & 509.24 & 1.36 \\
\hline 29 & 1233 & 562.16 & 570.00 & 554.45 & 1.37 & 554.19 & 1.42 & 570.46 & 0.08 & 560.69 & 0.26 \\
\hline 30 & 1333 & 600.00 & 605.31 & 595.28 & 0.79 & 594.46 & 0.92 & 619.39 & 2.35 & 614.80 & 1.58 \\
\hline $31^{\#}$ & 2222 & 556.30 & 556.60 & 550.48 & 1.05 & 550.07 & 1.12 & 555.97 & 0.06 & 551.55 & 0.85 \\
\hline 32 & 2223 & 588.00 & 602.46 & 592.71 & 0.00 & 591.95 & 0.00 & 605.34 & 0.49 & 605.33 & 0.49 \\
\hline 33 & 2233 & 640.00 & 668.21 & 654.93 & 0.00 & 654.18 & 0.00 & 676.93 & 1.36 & 661.90 & 0.02 \\
\hline 34 & 2333 & 674.67 & 678.00 & 658.63 & 2.37 & 657.83 & 2.50 & 688.90 & 1.61 & 721.38 & 6.43 \\
\hline 35 & 3333 & 722.00 & 741.99 & 722.49 & 0.00 & 720.72 & 0.18 & 763.96 & 3.04 & 783.94 & 5.81 \\
\hline
\end{tabular}

1 - Значком \# отмечены вещества, входящие в обучающую выборку. Значком * отмечены вещества, данные о которых встречаются только в [4], достоверность их не известна.

2 - Фреоны кодируются ключами, в которых цифры соответствуют атомам, связанным с атомом углерода:

$\mathrm{H} \equiv 0, \quad \mathrm{~F} \equiv 1, \quad \mathrm{Cl} \equiv 2, \quad \mathrm{Br} \equiv 3$.

3 - Экспериментальные данные приводятся по [4, 6, 8, 20 - 23].

4 - Вычислено нами. Относительная погрешность расчетных значений определялась как

$E=\left(\Delta F / F_{\min }\right) \cdot 100 \%$, где

$$
\Delta F=\left\{\begin{array}{ccc}
F_{\min }-F_{\text {calc }}, & \text { если } & F_{\text {calc }}<F_{\min }, \\
0, & \text { если } & F_{\text {min }}<F_{\text {calc }}<F_{\text {max }}, \\
F_{\text {calc }}-F_{\text {max }}, & \text { если } & F_{\text {calc }}>F_{\text {min }} .
\end{array}\right.
$$

5 - Веса вершин и ребер, использованные в расчете:

$a_{\mathrm{H}}=b_{\mathrm{H}}=1, \quad a_{\mathrm{F}}=0.751, \quad b_{\mathrm{F}}=2.49, \quad a_{\mathrm{Cl}}=0.822, \quad b_{\mathrm{Cl}}=2.767, \quad a_{\mathrm{Br}}=0.872, \quad b_{\mathrm{Br}}=2.848$.

Метод статистических испытаний при оценке дисперсии этих параметров привел к значениям $\Delta a=0.003, \Delta b=0.01$.

Имеет место линейная регрессия $T_{c}(R) \mathrm{c}$

$k=165.257, c=-464.331, r=0.996, \sigma=2.13$. 
Таблица 2

Экспериментальные и расчетные значения $P_{c}$, МПа фреонов метанового ряда

\begin{tabular}{|c|c|c|c|c|c|c|c|c|c|}
\hline \multirow[t]{2}{*}{ № ${ }^{1}$} & \multirow[t]{2}{*}{ Ключ ${ }^{2}$} & \multicolumn{2}{|c|}{ Эксперимент. данные } & \multicolumn{2}{|c|}{ Метод Сладкова $^{4}$} & \multicolumn{2}{|c|}{ Метод Лидерсена ${ }^{4}$} & \multicolumn{2}{|c|}{ Наш расчет $^{5}$} \\
\hline & & Min & Max & $P_{c}$ & $E, \%$ & $P_{c}$ & $E, \%$ & $P_{c}$ & $E \%$ \\
\hline $01^{\#}$ & 0000 & 4.53 & 4.64 & 4.13 & 8.96 & 5.05 & 9.14 & 4.65 & 0.13 \\
\hline $02^{\#}$ & 0001 & 5.86 & 5.88 & 6.57 & 11.81 & 5.51 & 5.92 & 5.89 & 0.29 \\
\hline 03 & 0002 & 6.49 & 6.68 & 6.76 & 1.19 & 6.50 & 0.00 & 6.36 & 2.00 \\
\hline 04 & 0003 & 5.22 & 8.61 & 6.80 & 0.00 & 8.45 & 0.00 & 7.27 & 0.00 \\
\hline 05 & 0011 & 5.39 & 5.84 & 6.83 & 18.29 & 5.12 & 5.10 & 5.58 & 0.00 \\
\hline $06^{*}$ & 0012 & 6.00 & 6.00 & 6.50 & 8.30 & 5.62 & 6.35 & 5.92 & 1.56 \\
\hline $07^{*}$ & 0013 & 6.11 & 6.11 & 6.43 & 5.21 & 6.86 & 12.34 & 6.43 & 5.25 \\
\hline $08^{\#}$ & 0022 & 6.08 & 6.17 & 6.61 & 7.22 & 5.91 & 2.84 & 6.28 & 1.89 \\
\hline $09 *$ & 0023 & 6.32 & 6.32 & 6.24 & 1.31 & 6.81 & 7.76 & 6.80 & 7.57 \\
\hline 10 & 0033 & 6.47 & 7.19 & 6.66 & 0.00 & 7.17 & 0.00 & 7.23 & 1.33 \\
\hline $11^{\#}$ & 0111 & 4.81 & 4.83 & 5.39 & 11.68 & 4.75 & 1.26 & 4.71 & 2.02 \\
\hline $12^{\#}$ & 0112 & 4.93 & 4.99 & 5.15 & 3.22 & 5.04 & 1.06 & 4.96 & 0.00 \\
\hline $13^{*}$ & 0113 & 5.17 & 5.17 & 5.18 & 0.11 & 5.91 & 14.20 & 5.24 & 1.22 \\
\hline $14^{\#}$ & 0122 & 5.16 & 5.19 & 5.13 & 0.60 & 5.21 & 0.47 & 5.22 & 0.56 \\
\hline $15^{*}$ & 0123 & 5.35 & 5.35 & 5.38 & 0.43 & 5.88 & 9.74 & 5.50 & 2.77 \\
\hline $16^{*}$ & 0133 & 5.49 & 5.49 & 5.35 & 2.57 & 6.17 & 12.45 & 5.76 & 4.96 \\
\hline $17^{\#}$ & 0222 & 5.47 & 5.47 & 5.52 & 0.95 & 5.30 & 3.00 & 5.49 & 0.31 \\
\hline $18^{*}$ & 0223 & 5.60 & 5.60 & 5.34 & 4.77 & 5.81 & 3.67 & 5.78 & 3.08 \\
\hline $19 *$ & 0233 & 5.75 & 5.75 & 5.59 & 2.72 & 6.03 & 4.97 & 6.04 & 5.13 \\
\hline $20 *$ & 0333 & 5.91 & 5.91 & 5.49 & 7.14 & 6.09 & 3.04 & 6.29 & 6.33 \\
\hline $21^{\#}$ & 1111 & 3.74 & 3.81 & 3.69 & 1.38 & 4.26 & 12.13 & 3.76 & 0.00 \\
\hline $22^{\#}$ & 1112 & 3.86 & 3.92 & 3.81 & 1.29 & 4.45 & 13.74 & 3.93 & 0.27 \\
\hline $23^{\#}$ & 1113 & 3.95 & 3.97 & 3.97 & 0.00 & 5.09 & 28.28 & 4.08 & 2.79 \\
\hline $24^{\#}$ & 1122 & 4.12 & 4.14 & 4.07 & 1.12 & 4.57 & 10.32 & 4.11 & 0.16 \\
\hline $25^{\#}$ & 1123 & 4.25 & 4.34 & 4.16 & 2.08 & 5.07 & 17.13 & 4.27 & 0.00 \\
\hline $26^{\#}$ & 1133 & 4.26 & 4.33 & 4.34 & 0.15 & 5.32 & 23.22 & 4.41 & 1.66 \\
\hline $27^{\#}$ & 1222 & 4.37 & 4.41 & 4.32 & 1.08 & 4.63 & 4.99 & 4.30 & 1.35 \\
\hline $28^{*}$ & 1223 & 4.44 & 4.44 & 4.48 & 0.87 & 5.03 & 13.24 & 4.46 & 0.41 \\
\hline $29^{\#}$ & 1233 & 4.58 & 4.69 & 4.55 & 0.68 & 5.23 & 11.72 & 4.60 & 0.00 \\
\hline $30^{\#}$ & 1333 & 4.65 & 4.73 & 4.73 & 0.05 & 5.30 & 12.31 & 4.73 & 0.00 \\
\hline $31^{\#}$ & 2222 & 4.49 & 4.56 & 4.56 & 0.08 & 4.65 & 2.07 & 4.50 & 0.00 \\
\hline $32^{\#}$ & 2223 & 4.66 & 4.69 & 4.70 & 0.09 & 4.97 & 5.97 & 4.66 & 0.03 \\
\hline $33^{\#}$ & 2233 & 4.78 & 4.83 & 4.88 & 0.91 & 5.13 & 6.10 & 4.80 & 0.00 \\
\hline $34^{\#}$ & 2333 & 4.85 & 5.02 & 4.67 & 3.78 & 5.18 & 3.18 & 4.93 & 0.00 \\
\hline $35^{\#}$ & 3333 & 5.07 & 5.21 & 5.05 & 0.48 & 5.17 & 0.00 & 5.05 & 0.36 \\
\hline
\end{tabular}

$1-4$ - См. сноски к табл. 1.

5 - Веса вершин и ребер, использованные в расчете:

$$
a_{\mathrm{H}}=b_{\mathrm{H}}=1, \quad a_{\mathrm{F}}=0.621, \quad b_{\mathrm{F}}=3.305, \quad a_{\mathrm{Cl}}=0.64, \quad b_{\mathrm{Cl}}=3.685, \quad a_{\mathrm{Br}}=0.62, \quad b_{\mathrm{Br}}=4.595 .
$$

Имеет место линейная регрессия $P_{c}(R)$ с $k=1.0672, c=0.377, r=0.995, \sigma=0.01_{5}$.

Для сравнительного анализа методов расчета $T_{c}$ и $P_{c}$ все вещества разбивались на группы по надежности экспериментальных данных. Результаты сравнения сведены в табл. 3, 4. Для каждой группы указаны число входящих в нее соединений $n$ и относительная погрешность литературных экспериментальных данных $E_{\exp }=\frac{F_{\max }-F_{\min }}{F_{\min }} 100 \%$. Соединения, сведения о которых встречаются только в [4], отнесены в последние группы. Для сравнения приводятся средние $E_{\text {av }}$ и $E_{\max }$ максимальные относительные погрешности по группам данных. 
Таблица 3

Сравнение методов расчета $T_{c}$ для галогенпроизводных метана

\begin{tabular}{|c|c|c|c|c|c|}
\hline \multirow[t]{2}{*}{ Группы } & \multirow[t]{2}{*}{$E, \%$} & \multicolumn{3}{|c|}{ Метод } & \multirow{2}{*}{$\begin{array}{c}\text { Наши } \\
\text { расчеть }\end{array}$} \\
\hline & & Сладкова-1 & Сладкова-2 & Лидерсона & \\
\hline \multirow{2}{*}{$\begin{array}{l}\text { Группа А } n=18 \\
E_{\text {exp }}<1 \%\end{array}$} & $E_{\mathrm{av}}$ & 1.79 & 1.6 & 0.58 & 1.84 \\
\hline & $E_{\max }$ & 5.24 & 4.89 & 1.55 & 3.74 \\
\hline \multirow{2}{*}{$\begin{array}{l}\text { Группа Б } \\
\text { Bce } n=35\end{array}$} & $E_{\mathrm{av}}$ & 1.45 & 1.29 & 0.69 & 2.04 \\
\hline & $E_{\max }$ & 5.24 & 4.89 & 3.04 & 6.43 \\
\hline Привлекаемые экспер. данные & & $T_{\text {кип }, H}, \rho_{\text {кии ж }}$ & $T_{\text {кun }, \mathrm{H}}$ & $T_{\text {кun }, \mathrm{H}}$ & - \\
\hline
\end{tabular}

Таблица 4

Сравнение методов расчета $P_{c}$ для галогенпроизводных метана

\begin{tabular}{|c|c|c|c|c|}
\hline \multirow[t]{2}{*}{ Группы } & \multirow[t]{2}{*}{$E, \%$} & \multicolumn{2}{|c|}{ Метод } & \multirow{2}{*}{$\begin{array}{c}\text { Наши } \\
\text { расчеты }\end{array}$} \\
\hline & & Сладкова & Лидерсона & \\
\hline \multirow{2}{*}{$\begin{array}{l}\text { Группа А } n=7 \\
E_{\exp }<1 \%\end{array}$} & $E_{\mathrm{av}}$ & 6.20 & 7.57 & 1.05 \\
\hline & $E_{\max }$ & 11.67 & 28.28 & 2.79 \\
\hline \multirow{2}{*}{$\begin{array}{l}\text { Группа Б } n=16 \\
E_{\text {exp }}<3 \%\end{array}$} & $\overline{E_{\text {av }}}$ & 2.50 & 7.74 & 0.83 \\
\hline & $E_{\max }$ & 11.67 & 28.28 & 2.79 \\
\hline \multirow{2}{*}{$\begin{array}{l}\text { Группа В } \\
\text { Bce } n=35\end{array}$} & $\overline{E_{\text {av }}}$ & 3.09 & 6.90 & 1.53 \\
\hline & $E_{\max }$ & 18.29 & 28.28 & 7.57 \\
\hline Привлекаемые экспер. данные & & $T_{c}$ & - & - \\
\hline
\end{tabular}

Преимущество применения ИП для вычисления $P_{c}$ очевидно. Для $T_{c}$ ситуация несколько хуже, однако, предлагаемый нами метод не требует привлечения дополнительных экспериментальных данных, как в методах Сладкова и Лидерсена, что резко сужает возможности этих методов применительно к веществам, для которых нужные экспериментальные данные еще не измерены, не говоря уже о ситуации, когда не синтезированы еще сами вещества.

Этап 3. Далее рассматриваем галогензамещенные этана. В дополнение к уже подобранным параметрам необходимы еще два: $a_{\mathrm{C}}$ и $b_{\mathrm{C}} \equiv b_{\text {с-с }}$. Выразим ИП $R$ в общем виде через $a_{\text {с и }}$ $b_{\mathrm{C}}$, считая их переменными, и через ранее найденные параметры атомов

$$
A \equiv\left\{a_{i}\right\}=\left(a_{\mathrm{H}}, a_{\mathrm{F}}, a_{\mathrm{Cl}}, a_{\mathrm{Br}}\right) \quad \text { и } \quad \text { связей }
$$

$B \equiv\left\{b_{i}\right\}=\left(b_{\mathrm{H}}, b_{\mathrm{F}}, b_{\mathrm{Cl}}, b_{\mathrm{Br}}\right)$, считая их константами:

$$
R=a_{\mathrm{C}} \Pi \sum_{i=1}^{6} \frac{b_{i}}{a_{i}}+b_{\mathrm{C}} \Pi+\Pi \sum_{i=1}^{3} \sum_{j=4}^{6} \frac{b_{i}}{a_{i}} \frac{b_{j}}{a_{j}}, \quad \Pi=\prod_{i=1}^{6} a_{i} .
$$

Выпишем значения, которые известны до подбора $a_{\text {С }}$ и $b_{\mathrm{C}}$ для некоторого $l$-го соединения:

$$
S_{1}^{(l)}=\Pi^{(l)} \sum_{i=1}^{6} \frac{b_{i}^{(l)}}{a_{i}^{(l)}}, \quad S_{2}^{(l)}=\Pi^{(l)}, \quad S_{3}^{(l)}=\Pi^{(l)} \sum_{i=1}^{3} \sum_{j=4}^{6} \frac{b_{i}^{(l)}}{a_{i}^{(l)}} \frac{b_{j}^{(l)}}{a_{j}^{(l)}}
$$

В предположении, что рассматриваемое свойство вещества $F$ зависит линейно от ИП $R$, в чем на примере $T_{c}$ и $P_{c}$ мы уже убедились из приведенные выше регрессионных уравнений и рис. 1 и 2, получим:

$F^{(l)}=k_{1} S_{1}^{(l)}+k_{2} S_{2}^{(l)}+k_{3} S_{3}^{(l)}+C, k_{1}=k a_{\mathrm{C}}, k_{2}=k b_{\mathrm{C}}, k_{3}=k$.

Таким образом, для нахождения параметров $a_{\text {С }}$ и $b_{\text {С }}$ достаточно воспользоваться стандартными программами линейного регрессионного анализа.

Для оценки устойчивости решения из 19 соединений с известными $T_{c}$ и $P_{c} 16$ соединений выбирались для построения регрессионного уравнения, а остальные 3 оставались для контроля прогнозирующей способности. Выполненные расчеты при 20 разных выборках опорных соединениях показали устойчивость искомых коэффициентов относительно выбора обучающих соединений. В дальнейшем из всех уравнений было выбрано для расчета параметров уравнение с наилучшими прогнозирующими и статистическими характеристиками. Результаты расчетов представлены в табл. 5 и 6. 
Таблица 5

Экспериментальные и расчетные значения $T_{c}, K$ фреонов этанового ряда

\begin{tabular}{|c|c|c|c|c|c|c|c|c|}
\hline \multirow[t]{2}{*}{ № ${ }^{1}$} & \multirow[t]{2}{*}{ Ключ ${ }^{2}$} & \multirow{2}{*}{$\begin{array}{l}\text { Экспер. } \\
\text { данные }^{3}\end{array}$} & \multicolumn{2}{|c|}{ Метод Сладкова- $2^{4}$} & \multicolumn{2}{|c|}{ Метод Лидерсена ${ }^{4}$} & \multicolumn{2}{|c|}{ Наш расчет $^{5}$} \\
\hline & & & $T_{c}$ & $E, \%$ & $T_{c}$ & $E, \%$ & $T_{c}$ & $E, \%$ \\
\hline $01^{\#}$ & $111-112$ & 352.9 & 368.6 & 4.37 & 353.2 & 0.08 & 358.5 & 1.51 \\
\hline 02 & $111-122$ & 418.6 & 434.5 & 3.79 & 418.6 & 0.00 & 415.1 & 0.83 \\
\hline $03^{\#}$ & $112-112$ & 418.8 & 434.5 & 3.79 & 418.4 & 0.10 & 415.2 & 0.87 \\
\hline $04^{\#}$ & $112-122$ & 487.0 & 501.2 & 2.86 & 485.2 & 0.37 & 477.4 & 1.98 \\
\hline $05^{\#}$ & $122-122$ & 551.0 & 568.0 & 3.09 & 552.4 & 0.25 & 545.7 & 0.96 \\
\hline $06^{\#}$ & $111-111$ & 292.8 & 308.6 & 3.90 & 293.8 & 0.34 & 307.0 & 4.86 \\
\hline $07^{\#}$ & $000-112$ & 410.0 & 416.6 & 1.60 & 411.6 & 0.39 & 403.2 & 1.66 \\
\hline $08^{\#}$ & $002-022$ & 602.0 & 606.3 & 0.72 & 601.6 & 0.07 & 633.1 & 5.17 \\
\hline $09^{\#}$ & 000-111 & 346.2 & 358.7 & 3.62 & 354.8 & 2.48 & 347.4 & 0.36 \\
\hline 10 & $000-022$ & 523.0 & 488.7 & 6.55 & 525.6 & 0.49 & 510.8 & 2.32 \\
\hline $11^{\#}$ & $002-002$ & 561.0 & 527.5 & 5.97 & 561.1 & 0.02 & 572.4 & 2.04 \\
\hline $12^{\#}$ & 000-011 & 386.6 & 395.8 & 2.39 & 394.1 & 1.94 & 385.5 & 0.54 \\
\hline $13^{\#}$ & $000-003$ & 503.8 & 492.7 & 2.21 & 506.9 & 0.62 & 475.9 & 5.53 \\
\hline $14^{\#}$ & $000-002$ & 460.4 & 452.7 & 1.68 & 459.8 & 0.13 & 445.8 & 3.18 \\
\hline $15^{\#}$ & $000-001$ & 375.3 & 358.6 & 4.44 & 378.7 & 0.91 & 384.4 & 2.43 \\
\hline $16^{\#}$ & $000-000$ & 305.4 & 285.1 & 7.62 & 304.8 & 0.20 & 318.5 & 4.29 \\
\hline $17^{\#}$ & $113-123$ & 563.0 & 572.5 & 1.69 & 565.7 & 0.48 & 552.0 & 1.96 \\
\hline $18^{\#}$ & $113-113$ & 487.6 & 500.2 & 2.54 & 492.1 & 0.92 & 483.0 & 0.94 \\
\hline $19^{6}$ & 003-003 & 583.0 & 592.0 & 1.54 & - & - & $(674.9)$ & $(11.1)$ \\
\hline \multicolumn{4}{|c|}{$E_{\mathrm{av}}, \%$} & 3.49 & & 0.54 & & 2.30 \\
\hline \multicolumn{4}{|c|}{$E_{\max }, \%$} & 7.62 & & 2.48 & & 5.53 \\
\hline
\end{tabular}

1 - 4 См. сноски к табл. 1. В литературных экспериментальных данных почти нет расхождений.

5 - Расчет производился с параметрами $a_{c}=4.012, b_{c}=-0.363$; дисперсии параметров: $\Delta a_{c}=0.08, \Delta b_{c}=0.02$. Остальные параметры - прежние (табл. 1). Имеет место линейная регрессия $T_{c}(R)$ с $k=16.2816, c=-214.07, r=0.992, \sigma=2.01$.

6 - Экспериментальное значение $T_{c}$ для этого соединения встречается только в [23]. Для него не известна $T_{\kappa и n, н}$, и $T_{c}$ по Лидерсену вычислить невозможно. Есть сомнения в надежности приведенного экспериментального значения. Оно в конечном итоге было исключено из окончательного расчета предлагаемым нами методом.

Таблица 6

Экспериментальные и расчетные значения $P_{c}$, МПа фреонов этанового ряда

\begin{tabular}{|c|c|c|c|c|c|c|c|}
\hline \multirow[t]{2}{*}{ № ${ }^{1}$} & \multirow[t]{2}{*}{ Ключ $^{2}$} & \multicolumn{2}{|c|}{ Экспер. данные ${ }^{3}$} & \multicolumn{2}{|c|}{ Метод Лидерсена } & \multicolumn{2}{|c|}{ Наш расчет $^{5}$} \\
\hline & & Min & Max & $P_{c}$ & $E, \%$ & $P_{c}$ & $E, \%$ \\
\hline $01^{\#}$ & $111-112$ & 3.16 & 3.19 & 3.23 & 1.28 & 3.10 & 1.90 \\
\hline 02 & $111-122$ & 3.30 & & 3.28 & 0.48 & 3.19 & 3.20 \\
\hline $03^{\#}$ & $112-112$ & 2.26 & 3.33 & 3.28 & 0.00 & 3.19 & 3.18 \\
\hline $04^{\#}$ & $112-122$ & 3.39 & 3.41 & 3.32 & 2.11 & 3.29 & 2.95 \\
\hline $05^{\#}$ & $122-122$ & 3.33 & 3.41 & 3.34 & 0.00 & 3.40 & 0.00 \\
\hline $06^{\#}$ & $111-111$ & 2.88 & 2.98 & 3.16 & 5.98 & 3.01 & 1.04 \\
\hline $07^{\#}$ & $000-112$ & 4.12 & 4.14 & 4.26 & 3.06 & 4.13 & 0.00 \\
\hline $08^{6}$ & $002-022$ & 4.15 & & 4.48 & 7.93 & 5.22 & 25.9 \\
\hline $09^{\#}$ & $000-111$ & 3.76 & 4.11 & 4.05 & 0.00 & 3.98 & 0.00 \\
\hline $10^{\#}$ & $000-022$ & 5.06 & & 4.99 & 1.38 & 5.07 & 0.18 \\
\hline $11^{\#}$ & $002-002$ & 5.37 & & 4.87 & 9.22 & 5.68 & 5.83 \\
\hline $12^{\#}$ & 000-011 & 4.49 & 4.50 & 4.46 & 0.67 & 4.65 & 3.33 \\
\hline $13^{\#}$ & 000-003 & 6.23 & & 6.77 & 8.65 & 5.85 & 5.58 \\
\hline $14^{\#}$ & 000-002 & 5.27 & 5.39 & 5.43 & 0.75 & 5.41 & 0.38 \\
\hline $15^{\#}$ & $000-001$ & 4.72 & 5.02 & 4.86 & 0.00 & 5.13 & 2.19 \\
\hline 16 & $000-000$ & 4.88 & 4.93 & 4.83 & 0.99 & 4.94 & 0.20 \\
\hline 17 & 113-123 & 3.52 & 4.06 & 3.69 & 0.00 & 3.47 & 1.40 \\
\hline $18^{\#}$ & $113-113$ & 3.36 & 3.49 & 3.73 & 6.91 & 3.37 & 0.00 \\
\hline $19^{6}$ & 003-003 & 7.14 & & 5.92 & 17.0 & 6.62 & 7.19 \\
\hline \multicolumn{5}{|c|}{$E_{\mathrm{av}}, \%$} & 2.44 & & 1.84 \\
\hline \multicolumn{5}{|c|}{$E_{\max }, \%$} & 9.22 & & 5.83 \\
\hline
\end{tabular}

1 - 4 См. сноски к табл. 1.

5 - Расчет производился с параметрами $a_{c}=5.846, b_{c}=11.034$. Остальные параметры - прежние (табл. 2). Имеет место линейная регрессия $P_{c}(R)$ с $k=0.0662, c=1.293$

6 - Эти вещества исключены из рассмотрения. Упоминаются в литературе только один раз, достоверность их не известна. В обоих методах дают большую погрешность. 
Обращает на себя внимание тот факт, что ИП хорошо описывает свойства изомеров, например, соединения с номерами 2, 3 и 10, 11 в табл. 5 и 6. Эту закономерность можно исследовать в общем виде. Рассмотрим два изомера $C X_{3}-C X Y_{2}$ (1) и $C X_{2} Y-C X_{2} Y(2)$. Для них

$$
\begin{aligned}
R_{1} & =3 b_{\mathrm{X}}^{2} a_{\mathrm{X}}^{2} a_{\mathrm{Y}}^{2}+6 b_{\mathrm{X}} b_{\mathrm{Y}} a_{\mathrm{X}}^{3} a_{\mathrm{Y}}+S, \\
R_{2} & =4 b_{\mathrm{X}}^{2} a_{\mathrm{X}}^{2} a_{\mathrm{Y}}^{2}+4 b_{\mathrm{X}} b_{\mathrm{Y}} a_{\mathrm{X}}^{3} a_{\mathrm{Y}}+b_{\mathrm{Y}}^{2} a_{\mathrm{X}}^{4}+S, \\
S & =a_{\mathrm{C}}\left(4 b_{\mathrm{X}} a_{\mathrm{X}}^{3} a_{\mathrm{Y}}^{2}+2 b_{\mathrm{Y}} a_{\mathrm{X}}^{4} a_{\mathrm{Y}}\right)+b_{\mathrm{C}} a_{\mathrm{X}}^{4} a_{\mathrm{Y}}^{2}, \\
R_{2} & -R_{1}=b_{\mathrm{X}}^{2} a_{\mathrm{X}}^{2} a_{\mathrm{Y}}^{2}+b_{\mathrm{Y}}^{2} a_{\mathrm{X}}^{4}-2 b_{\mathrm{X}} b_{\mathrm{Y}} a_{\mathrm{X}}^{3} a_{\mathrm{Y}}=a_{\mathrm{X}}^{2}\left(b_{\mathrm{X}} a_{\mathrm{Y}}-b_{\mathrm{Y}} a_{\mathrm{X}}\right)^{2} .
\end{aligned}
$$

Таким образом, ИП для изомеров со структурой (2) всегда больше либо равен ИП для изомеров со структурой (1), равенство же выполняется при условии $b_{\mathrm{X}} / b_{\mathrm{Y}}=a_{\mathrm{X}} / a_{\mathrm{Y}}$. Это обстоятельство можно использовать для подбора параметров. Из рассмотренного случая следуют два вывода:

1) на этапе 1 после подтверждения возможности применения данного ТИ можно использовать особенности изомеров для получения дополнительных ограничений на параметры;

2) с помощью ИП можно описывать $T_{c}$ и $P_{c}$, но нельзя описать $V_{c}$, поскольку при исследовании этого свойства, как будет видно из дальнейшего, наблюдается обратный эффект.

Вычисленные нами значения $T_{c}$ и $P_{c}$ для всех 210 фреонов этанового ряда приведены в $[24,25]$.

Этап 4. Расчет $T_{c}$ и $P_{c}$ для галогенпроизводных пропана. Новые параметры не требуются. Задача состоит лишь в том, чтобы найти коэффициенты корреляционного уравнения и оценить его прогнозирующую способность. Если учесть, что для статистически значимого уравнения число веществ должно в 4-5 раз превосходить число параметров, то обучающая выборка должна содержать не менее 8 соединений.

В нашем распоряжении было всего 9 соединений с известными свойствами, но не известной достоверности. Все они были использованы для получения регрессионного уравнения для расчета $T_{c}$. Когда станут доступными более точные экспериментальные данные и для большего числа соединений, желательно уточнить регрессионное уравнение. Расхождения в литературных экспериментальных данных $P_{c}$ и $V_{c}$ для галогензамещанных пропана вообще не позволяют построить хоть сколько-нибудь достоверное уравнение. Результаты расчетов по $T_{c}$ представлены в табл. 7.

\begin{tabular}{|c|c|c|c|c|c|c|}
\hline \multirow[t]{2}{*}{ № ${ }^{\mathrm{I}}$} & \multirow[t]{2}{*}{ Ключ $^{2}$} & \multirow{2}{*}{$\begin{array}{l}\text { Экспер. } \\
\text { данные }\end{array}$} & \multicolumn{2}{|c|}{ Метод Лидерсена } & \multicolumn{2}{|c|}{ Наш расчет ${ }^{5}$} \\
\hline & & & $T_{c}$ & $E, \%$ & $T_{c}$ & $E, \%$ \\
\hline $01^{\#}$ & 111-11-222 & 505.0 & 502.9 & 0.42 & 476.5 & 5.70 \\
\hline $02^{\#}$ & 111-12-112 & 451.0 & 447.7 & 0.73 & \begin{tabular}{|l|}
431.5 \\
\end{tabular} & 4.31 \\
\hline $03^{\#}$ & $111-11-112$ & 395.0 & 394.2 & 0.20 & 389.0 & 1.32 \\
\hline $04^{\#}$ & 111-11-111 & 345.0 & 343.6 & 0.41 & 352.3 & 2.11 \\
\hline $05^{\#}$ & $000-00-000$ & 369.8 & 372.2 & 0.65 & 396.9 & 7.32 \\
\hline $06^{\# 6}$ & 000-11-111 & 380.1 & & & 407.3 & 7.15 \\
\hline $07^{\#}$ & $000-00-002$ & 503.0 & 500.9 & 0.42 & 474.1 & 5.75 \\
\hline $08^{\#}$ & $002-02-002$ & 651.0 & 650.6 & 0.06 & 666.7 & 2.42 \\
\hline $09^{\#}$ & $000-02-002$ & 577.0 & 572.3 & 0.82 & 582.1 & 0.88 \\
\hline \multicolumn{4}{|c|}{$E_{\mathrm{av}}, \%$} & 0.46 & & 4.11 \\
\hline \multicolumn{4}{|c|}{$E_{\max }, \%$} & 0.82 & & 7.32 \\
\hline
\end{tabular}

Таблица 7

Экспериментальные и расчетные значения $T_{c}$, $K$ фреонов пропанового ряда

$1-4$ См. сноски к табл. 1.

5 - Расчет производился с такими же параметрами, как и для этановых фреонов.

6 - Для этого соединения не известна $T_{\text {кии, }, ~}$ и $T_{c}$ по Лидерсену вычислить невозможно.

Вычисленные нами значения $T_{c}$ для всех 2100 фреонов пропанового ряда приведены в [24, 25].

\section{8. Параметризация и расчет $V_{c}$ фреонов}

Критический объем зависит от структуры молекулы практически аддитивно, что и обусловило появление большого числа инкрементных методов расчета [7]. При этом, естественно, ни один из них не отражает существенной разницы между значениями $V_{c}$ для изомеров $\mathrm{CH}_{2} \mathrm{Cl}_{-} \mathrm{CH}_{2} \mathrm{CL}$ и $\mathrm{CH}_{3}-\mathrm{CHCL}_{2}$, которые составляют соответственно 220 и $240 \mathrm{~cm}^{3} \cdot$ моль $^{-1}$. Выше было показано, что ИП также не отражает это различие между изомерами. Поэтому велся поиск других ТИ.

Обратимся к введенному нами индексу ${ }^{2} \tilde{\chi}$ (1). Поскольку для галогензамещенных алканов в индексе ${ }^{2} \tilde{\chi}$ параметры вершин и ребер встречаются только в виде произведения, то их можно заменить одним параметром. Рассмотрим изомеры $\mathrm{CX}_{3}-\mathrm{CXY}_{2}$ (1) и $\mathrm{CX}_{2} \mathrm{Y}-\mathrm{CX}_{2} \mathrm{Y}(2)$. Для них при $x=a_{x} b_{x}, \quad y=a_{y} b_{y}$ :

$$
\begin{aligned}
& { }^{2} \tilde{\chi}_{1}=a_{\mathrm{C}}^{2} b_{\mathrm{C}}(4 x+2 y)+a_{\mathrm{C}}\left(y^{2}+2 x y+3 x^{2}\right), \\
& { }^{2} \tilde{\chi}_{2}=a_{\mathrm{C}}^{2} b_{\mathrm{C}}(4 x+2 y)+a_{\mathrm{C}}\left(2 x^{2}+4 x y\right), \\
& { }^{2} \tilde{\chi}_{1}-{ }^{2} \tilde{\chi}_{2}=a_{\mathrm{C}}\left(y^{2}-2 x y+x^{2}\right)=a_{\mathrm{C}}(x-y)^{2} \geq 0 .
\end{aligned}
$$

Казалось бы, этот индекс мог бы подойти для описания $V_{c}$, однако, равенство, которое имеет место для соединений $\mathrm{CF}_{3}-\mathrm{CFCl}_{2}$ и $\mathrm{CF}_{2} \mathrm{Cl}-\mathrm{CF}_{2} \mathrm{Cl}$, может выполняться только при $\mathrm{X}=\mathrm{Y}$, что неприемлемо. Поэтому возникла потребность модифицировать индекс ${ }^{2} \tilde{\chi}$ по аналогии с ИП, т. е. умножать произведение весов смежных ребер не на 
веса вершин, которые они покрывают, а на веса вершин, не покрываемых этими ребрами, а именно:

$$
{ }^{2} \tilde{\tilde{\chi}}=\sum_{i, j, k-a d j}\left(b_{i j} b_{j k} \prod_{\substack{l=1 \\ l \neq i, j, k}}^{p} a_{l}\right)=\prod_{i=1}^{p} a_{i} \cdot \sum_{i, j, k-a d j} \frac{b_{i j} b_{j k}}{a_{i} a_{j} a_{k}},
$$

где $p$ - число вершин, а суммирование ведется по всем парам смежных ребер. Для этого инварианта

$$
{ }^{2} \tilde{\tilde{\chi}}_{1}-{ }^{2} \tilde{\tilde{\chi}}_{2}=a_{\mathrm{C}} a_{\mathrm{X}}^{2}\left(b_{\mathrm{X}} a_{\mathrm{Y}}-b_{\mathrm{Y}} a_{\mathrm{X}}\right)^{2},
$$

т. е. равенство выполняется при условии

$$
b_{\mathrm{X}} / b_{\mathrm{Y}}=a_{\mathrm{X}} / a_{\mathrm{Y}} \text {. }
$$

Отсюда получаем дополнительные зависимости между параметрами:

$$
\frac{b_{\mathrm{Cl}}}{b_{\mathrm{F}}}=\frac{a_{\mathrm{Cl}}}{a_{\mathrm{F}}}, \quad \frac{b_{\mathrm{Cl}}}{b_{\mathrm{H}}} \neq \frac{a_{\mathrm{Cl}}}{a_{\mathrm{H}}} .
$$

Расчеты $V_{c}$ выполнялись аналогично расчетам $T_{c}$ и $P_{c}$ с учетом (43). Отметим, что на этапе 3 (этановый ряд) для вычисления $a_{\mathrm{C}}$ и $b_{\mathrm{C}}$ использовалось выражение

$$
{ }^{2} \tilde{\tilde{\chi}}\left(\mathrm{CX}_{1} \mathrm{X}_{2} \mathrm{X}_{3}-\mathrm{CX}_{4} \mathrm{X}_{5} \mathrm{X}_{6}\right)=a_{\mathrm{C}} \prod_{i=1}^{6} a_{i} \cdot \sum_{i, C, j-a d j} \frac{b_{i}}{a_{i}} \frac{b_{j}}{a_{j}}+b_{\mathrm{C}} \prod_{i=1}^{6} a_{i} \cdot \sum_{i=1}^{6} \frac{b_{i}}{a_{i}} \cdot
$$

\begin{tabular}{|c|c|c|c|c|c|c|c|c|c|c|c|}
\hline \multirow[t]{2}{*}{ №1 ${ }^{1}$} & \multirow[t]{2}{*}{ Ключ $^{2}$} & \multicolumn{2}{|c|}{ Эксперимент. данные } & \multicolumn{2}{|c|}{ Метод Сладкова ${ }^{4}$} & \multicolumn{2}{|c|}{ Метод Воулеса $^{4}$} & \multicolumn{2}{|c|}{ Метод Лидерсена $^{4}$} & \multicolumn{2}{|c|}{ Наш расчет $^{5}$} \\
\hline & & Min & Max & $V_{c}$ & $E, \%$ & $V_{c}$ & $E, \%$ & $V_{c}$ & $E, \%$ & $V_{c}$ & $E, \%$ \\
\hline $01^{\#}$ & 0000 & 98.40 & 117.42 & 101.97 & 0.00 & 91.00 & 7.52 & 93.00 & 3.46 & 98.13 & 0.28 \\
\hline $02^{\#}$ & 0001 & 105.00 & 114.97 & 109.85 & 0.00 & 107.10 & 0.00 & 113.00 & 0.00 & 110.13 & 0.00 \\
\hline $03^{\#}$ & 0002 & 126.51 & 139.10 & 136.09 & 0.00 & 137.50 & 0.00 & 144.00 & 3.87 & 138.57 & 0.00 \\
\hline $04^{\#}$ & 0003 & 150.00 & 158.76 & 149.56 & 0.29 & 156.00 & 0.00 & 165.00 & 4.16 & 149.91 & 0.06 \\
\hline $05^{\#}$ & 0011 & 121.00 & 122.40 & 120.36 & 0.53 & 123.20 & 0.66 & 131.00 & 7.11 & 121.77 & 0.00 \\
\hline $06^{*}$ & 0012 & 154.41 & 154.41 & 149.22 & 3.36 & 153.60 & 0.52 & 162.00 & 4.92 & 150.92 & 2.26 \\
\hline $07 *$ & 0013 & 167.63 & 167.63 & 164.80 & 1.69 & 172.10 & 2.67 & 183.00 & 9.17 & 164.45 & 1.90 \\
\hline $08^{\#}$ & 0022 & 178.00 & 183.00 & 172.90 & 2.86 & 184.00 & 0.56 & 193.00 & 5.62 & 181.89 & $0 / 00$ \\
\hline $09^{\#}$ & 0023 & 193.33 & 194.00 & 198.26 & 2.20 & 202.50 & 2.40 & 214.00 & 10.35 & 196.31 & 1.19 \\
\hline $10^{\#}$ & 0033 & 206.83 & 208.00 & 201.36 & 2.64 & 221.00 & 6.29 & 235.00 & 13.05 & 205.81 & 0.49 \\
\hline $11^{\#}$ & 0111 & 132.40 & 133.10 & 130.86 & 1.16 & 139.30 & 4.68 & 145.00 & 8.99 & 133.00 & 0.00 \\
\hline $12^{\#}$ & 0112 & 160.96 & 168.20 & 164.95 & 0.00 & 169.70 & 0.93 & 176.00 & 4.85 & 162.83 & 0.00 \\
\hline $13 *$ & 0113 & 174.54 & 174.54 & 183.60 & 5.19 & 188.20 & 7.83 & 197.00 & 12.87 & 178.65 & 2.35 \\
\hline $14^{\#}$ & 0122 & 194.00 & 197.00 & 202.20 & 1.60 & 200.10 & 1.60 & 207.00 & 5.15 & 194.52 & 0.00 \\
\hline $15^{*}$ & 0123 & 208.47 & 208.47 & 216.35 & 3.78 & 218.60 & 4.86 & 228.00 & 9.37 & 211.37 & 1.39 \\
\hline $16^{*}$ & 0133 & 217.86 & 217.86 & 231.66 & 6.33 & 237.10 & 8.83 & 249.00 & 14.29 & 223.27 & 2.48 \\
\hline $17^{\#}$ & 0222 & 216.11 & 239.00 & 222.22 & 0.00 & 230.50 & 0.00 & 238.00 & 0.00 & 228.18 & 0.00 \\
\hline 18 & 0223 & 230.03 & 240.03 & 248.92 & 3.87 & 249.00 & 3.90 & 259.00 & 8.25 & 246.14 & 2.65 \\
\hline $19 *$ & 0233 & 250.94 & 250.94 & 264.31 & 5.33 & 267.50 & 6.60 & 280.00 & 11.58 & 258.83 & 3.14 \\
\hline 20 & 0333 & 255.59 & 263.00 & 280.33 & 6.78 & 286.00 & 9.00 & 301.00 & 14.87 & 265.87 & 1.12 \\
\hline $21^{\#}$ & 1111 & 139.75 & 147.00 & 146.60 & 0.00 & 155.40 & 6.01 & 153.00 & 4.29 & 143.78 & 0.00 \\
\hline $22^{\#}$ & 1112 & 174.42 & 180.00 & 185.61 & 3.21 & 185.80 & 3.33 & 184.00 & 2.29 & 174.27 & 0.09 \\
\hline $23^{\#}$ & 1113 & 193.39 & 200.00 & 200.09 & 0.05 & 204.30 & 2.22 & 205.00 & 2.59 & 192.44 & 0.49 \\
\hline $24^{\#}$ & 1122 & 208.79 & 217.10 & 219.46 & 1.13 & 216.20 & 0.00 & 215.00 & 0.00 & 206.65 & $1 / 03$ \\
\hline $25^{\#}$ & 1123 & 223.16 & 232.00 & 236.96 & 2.22 & 234.70 & 1.21 & 236.00 & 1.79 & 226.00 & 0.00 \\
\hline $26^{\#}$ & 1133 & 242.16 & 253.00 & 250.69 & 0.00 & 253.20 & 0.08 & 257.00 & 1.65 & 240.44 & 0.71 \\
\hline $27^{\#}$ & 1222 & 240.91 & 248.00 & 251.03 & 1.26 & 246.60 & 0.00 & 246.00 & 0.00 & 241.04 & 0.00 \\
\hline $28 *$ & 1223 & 258.30 & 258.30 & 264.91 & 2.56 & 265.10 & 2.63 & 267.00 & 3.37 & 261.66 & 1.30 \\
\hline $29^{\#}$ & 1233 & 272.09 & 283.00 & 282.62 & 0.00 & 283.60 & 0.22 & 288.00 & 1.84 & 277.05 & 0.00 \\
\hline 30 & 1333 & 283.51 & 300.00 & 291.88 & 0.00 & 302.10 & 0.74 & 309.00 & 3.17 & 286.63 & 0.00 \\
\hline 31 & 2222 & 276.00 & 277.65 & 279.95 & 0.83 & 277.00 & 0.00 & 277.00 & 0.00 & 277.58 & 0.00 \\
\hline 32 & 2223 & 284.54 & 291.00 & 292.83 & 0.64 & 295.50 & 1.58 & 298.00 & 2.46 & 299.53 & 3.00 \\
\hline 33 & 2233 & 305.94 & 311.00 & 311.55 & 0.18 & 314.00 & 0.98 & 319.00 & 2.61 & 315.95 & 1.62 \\
\hline 34 & 2333 & 296.73 & 324.00 & 327.58 & 1.21 & 332.50 & 2.86 & 340.00 & 5.39 & 326.18 & 0.74 \\
\hline 35 & 3333 & 314.16 & 330.00 & 332.30 & 0.73 & 351.00 & 6.68 & 361.00 & 9.87 & 330.12 & 0.04 \\
\hline \multicolumn{5}{|c|}{$E_{\mathrm{av}}, \%$} & 1.79 & & 2.78 & & 5.52 & & 0.82 \\
\hline \multicolumn{5}{|c|}{$E_{\max }, \%$} & 6.78 & & 9.00 & & 14.9 & & 3.14 \\
\hline
\end{tabular}

Результаты вычисления $V_{c}$ для фреонов метанового и этанового рядов приведены табл. 8, 9 .

Таблица 8

Экспериментальные и расчетные значения $V_{c}, \mathrm{~cm}^{3} \cdot$ моль ${ }^{-1}$ фреонов метанового ряда

$1-4$-См. сноски к табл. 1.

5 - Веса вершин и ребер, использованные в расчете: $a_{H}=b_{H}=1, a_{F}=1.095, b_{F}=0.959$

$a_{c l}=1.165, b_{c l}=1.017, a_{B r}=1.003, b_{B r}=1.23$; дисперсии параметров: $\Delta a=\Delta b=0.003$.

Имеет место линейная регрессия $V_{c}(R)$ с $k=74.075, c=-346.32, r=0.999, \sigma=0.33$. 
Таблица 9

Экспериментальные и расчетные значения $V_{c}, c^{3} \cdot$ моль $^{-1}$ фреонов этанового ряда

\begin{tabular}{|c|c|c|c|c|c|c|c|c|c|}
\hline \multirow[t]{2}{*}{ № ${ }^{1}$} & \multirow[t]{2}{*}{ Ключ ${ }^{2}$} & \multicolumn{2}{|c|}{ Экспер. данные } & \multicolumn{2}{|c|}{ Метод Воулеса $^{4}$} & \multicolumn{2}{|c|}{ Метод Лидерсена } & \multicolumn{2}{|c|}{ Наш расчет $^{5}$} \\
\hline & & Min & Max & $V_{c}$ & $E, \%$ & $V_{c}$ & $E, \%$ & $V_{c}$ & $E, \%$ \\
\hline $01^{\#}$ & $111-112$ & 231.5 & 252 & 275.0 & 9.13 & 261 & 3.57 & 257.3 & 2.09 \\
\hline $02^{\#}$ & $111-122$ & 294 & & 305.4 & 3.88 & 292 & 0.68 & 290.6 & 1.16 \\
\hline $03^{\#}$ & $112-112$ & 274.4 & 294 & 305.4 & 3.88 & 292 & 0.00 & 290.6 & 0.00 \\
\hline $04^{\#}$ & $112-122$ & 304 & 325 & 335.8 & 3.32 & 323 & 0.00 & 326.0 & 0.30 \\
\hline $05^{\#}$ & $122-122$ & 358.4 & 370 & 366.2 & 0.00 & 354 & 1.23 & 363.6 & 0.00 \\
\hline $06^{\#}$ & $111-111$ & 216.3 & 225 & 244.6 & 8.58 & 230 & 2.22 & 225.9 & 0.42 \\
\hline $07^{\#}$ & $000-112$ & 219 & 233 & 226.7 & 0.00 & 221 & 0.00 & 226.0 & 0.00 \\
\hline $08^{\#}$ & $002-022$ & 294 & & 287.5 & 2.21 & 293 & 0.34 & 284.4 & 3.26 \\
\hline $09^{\#}$ & 000-111 & 187.3 & 221 & 196.3 & 0.00 & 190 & 0.00 & 196.3 & 0.00 \\
\hline 10 & $000-022$ & 240 & & 241.0 & 0.42 & 244 & 1.67 & 239.4 & 0.25 \\
\hline $11^{\#}$ & $002-002$ & 220 & & 241.0 & 9.54 & 248 & 12.7 & 236.6 & 7.53 \\
\hline $12^{\#}$ & $000-011$ & 181 & 188 & 180.0 & 0.55 & 182 & 0.00 & 180.3 & 0.41 \\
\hline $13^{\#}$ & 000-003 & 215 & & 213.0 & 0.93 & 220 & 2.33 & 199.1 & 7.38 \\
\hline 14 & $000-002$ & 191.4 & 199 & 194.5 & 0.00 & 199 & 0.00 & 193.4 & 0.00 \\
\hline 15 & 000-001 & 158 & 169 & 164.1 & 0.00 & 168 & 0.00 & 165.6 & 0.00 \\
\hline $16^{\#}$ & $000-000$ & 140.6 & 148 & 148.0 & 0.00 & 150 & 1.35 & 152.1 & 2.78 \\
\hline $17^{\#}$ & $113-123$ & 346.6 & & 372.8 & 7.54 & 365 & 5.29 & 353.3 & 1.94 \\
\hline $18^{\#}$ & 113-113 & 319 & 328 & 342.4 & 4.39 & 334 & 1.83 & 316.2 & 0.87 \\
\hline $19^{\#}$ & $003-003$ & 242 & 260 & 278.0 & 6.92 & 290 & 11.5 & 246.4 & 0.00 \\
\hline \multicolumn{5}{|c|}{$E_{\mathrm{av}}, \%$} & 3.30 & & 2.66 & & 1.49 \\
\hline \multicolumn{5}{|c|}{$E_{\max }, \%$} & 9.54 & & 12.73 & & 7.54 \\
\hline
\end{tabular}

$1-4$ См. сноски к табл. 1.

5 - Расчет производился с параметрами $a_{\mathrm{C}}=1.75, b_{\mathrm{C}}=-0.781$; дисперсии параметров: $\Delta a_{\mathrm{C}}=0.06, \Delta b_{\mathrm{C}}=0.05$. Остальные параметры - прежние (табл. 8).

Имеет место линейная регрессия $V_{c}(R)$ с $k=74.075, c=-278.75, r=0.994, \sigma=1.38$.

Вычисленные нами значения $V_{c}$ для всех 210 фреонов этанового ряда приведены в [24, 25].

\section{9. Выводы}

Для количественного описания критических свойств фреонов предложен новый подход класса «структура - свойство» с использованием инвариантов полностью взвешенных графов. Он предполагает зависимость топологического описания молекул от изучаемого свойства. Сформулирован общий принцип построения топологических инвариантов полностью взвешенных графов для корреляций «структура свойство», а именно:

$$
I(G)=\sum_{G_{i}}\left[\left(\prod_{j \in E_{i}} b_{j}\right)\left(\prod_{k \in V_{i}^{\prime}} a_{k}\right)\right],
$$

где $G_{i}-$ всевозможные подграфы графа $G$ заданного вида, $E_{i}-$ множество ребер с весами $b_{j}$ этих подграфов, $V_{i}^{\prime}-$ множество вершин с весами $a_{k}$ подграфов $G_{i}^{\prime}=G \backslash G_{i}$. В соответствии с этим принципом предложены два новых инварианта ${ }^{2} \tilde{\chi}$ (1) и $R(G) \quad(10)$, примененных для расчета критических свойств $T_{c}, P_{c}$ и $V_{c}$ фреонов метанового, этанового и пропанового рядов.

Предложенный нами подход в отличие от инкрементных методов Лидерсена и Сладкова не предполагает привлечения каких-либо экспериментальных данных и закономерностей для расчета критических свойств. Для $P_{c}$ и $V_{c}$ получены заметно лучшие результаты по сравнению с методами Лидерсена, Воулеса и Сладкова. Что касается $T_{c}$, то результаты прогноза вполне приемлемы, учитывая также то обстоятельство, что не использовалось, как в других методах, экспериментальное значение $T_{\text {кии, }}$. Особо подчеркнем, что предлагаемый нами подход обеспечивает статистически надежную линейную зависимость всех критических свойств фреонов от значения индекса паросочетаний соответствующего молекулярного графа.

Вычислены более 2.5 тысяч ранее неизвестных значений критических свойств низших фреонов.

Результаты настоящего исследования были депонированы в 1988 году [24].

\section{Литература}

1. Balaban, A. T. Chemical Graphs. XXXIV. Five New Topological Indices for the Branching of Tree-like Graphs [Text] / A. T. Balaban // Theor. chim. Acta. - 1979. - Vol. 53, Issue 4. - P. 355-375.

2. Ловас, Л. Прикладные задачи теории графов. Теория паросочетаний в математике, физике, химии 
[Текст] / Л. Ловас, М. Пламмер; пер. с англ. - М.: Мир, 1998. $-653 \mathrm{c}$.

3. Химические приложения топологии и теории графов [Текст] / под ред. Р. Кинг, пер. с англ. - М.: Мир, 1987. $-560 \mathrm{c}$.

4. Перельштейн, И. И. Термодинамические и теплофизические свойства рабочих веществ холодильных машин и тепловых насосов [Текст] / И. И. Перельштейн, У. Б. Парушин: М.: Легкая промышленность, 1984. - 232 с.

5. Викторов, М. М. Методы вычисления физикохимических величин и прикладные расчеты [Текст] / М. М. Викторов. - Л.: Химия, 1977. - 360 с.

6. Рид, Р. Свойства газов и жидкостей [Текст] / Р. Рид, Дж. Праусниц, Т. Шервуд; пер. с англ. - Л.: Химия, $1982 .-592 \mathrm{c}$.

7. Филиппов, Л. П. Развитие методов расчета и прогнозирования свойств жидкостей [Текст] / Л. П. Филиппов, Е. В. Воробьева, А. Д. Охоцимский, Д. А. Толстунов. - Исследование строения, теплового движения и свойств жидкостей, М.: МГУ, 1986. C. $141-176$.

8. Морачевский, А. Г. Физико-химические свойства молекулярных неорганических соединений [Текст] / А. Г. Морачевский, И. Б. Сладков. - Л.: Химия, 1987. $-192 \mathrm{c}$

9. Jain D. V. S. Correlation Between Topological Features and Physicochemical Properties of Molecules [Text] / D. V. S. Jain, S. Singh, V. Gombar // Proc. Indian Acad. Sci. (Chem. Sci.). - 1984. - Vol. 93, Issue 6. - P. 927-945.

10. Кругляк, Ю. А. Установление связи "структуpa - свойство" для гетеросопряженных молекул с использованием инвариантов взвешенных графов и их информационных аналогов [Текст] : VII Всес. конф. / Ю. А. Кругляк, И. В. Передунова // Использование вычислительных машин в химических исследованиях и спектроскопии молекул. - Рига, 1986. - С. 115-117.

11. Knop, J. V. Chemical Graph Theory. II. On the Graph Theoretical Polynomials of Conjugated Structures [Text] / J. V. Knop, N. Trinajstic // Intern. J. Quantum Chem., Quantum Chem. Symp. - 1980. - Vol. 18, Issue S14. P. 503-520. doi: 10.1002/qua.560180853

12. Godsil, C. D. Some Remarks on the Matching Polynomial and Its Zeros [Text] / C. D. Godsil, I. Gutman // Croat. Chem. Acta. - 1981. - Vol. 54, Issue 1. - P. 53-59.

13. Godsil, C. D. Matchings and Walks in Graphs [Text] / C. D. Godsil // J. Graph Theory. - 1981. - Vol. 5, Issue 3. - P. 285-297. doi: 10.1002/jgt.3190050310

14. Hosoya, H. Operator Technique for Obtaining the Recursion Formulae of Characteristic and Matching Polynomials as Applied to Polyhex Graphs [Text] / H. Hosoya, N. J. Ohkami // Comput. Chem. - 1983. - Vol. 4, Issue 4. P. 585-593. doi: 10.1002/jcc.540040418

15. Hosoya H. Topological Index. A Newly Proposed Quantity Characterizing the Topological Nature of Structural Isomers of Saturated Hydrocarbons [Text] / H. Hosoya Bull. Chem. Soc. Japan. - 1971. - vol. 44, no. 9, pp. 2332 - 2339.

16. Farrel, E. J. An Introduction to Matching Polynomials [Text] / E. J. Farrel // J. Combinatorial Theory. 1979. - Vol. 27, Issue 1. - P. 75-86 doi: 10.1016/0095-8956(79)90070-4

17. Gutman, I. Topological Properties of Benzoid Systems. XXIX. On Hosoya's Topological Index [Text] / I. Gutman, A. Shalabi, Z. Naturforsch. - 1984. - Vol. 39a. P. 797-799.

18. Gutman, I. Graph Theory and Molecular Orbitals, 19. Nonparametric Resonance Energies of Arbitrary Cojugated Systems [Text] / I. Gutman, M. Milun, N. Trinajstic // J. Amer. Chem. Soc. - 1977. - Vol. 99, Issue 6. - P. 1692-1703. doi: $10.1021 / \mathrm{ja} 00448 \mathrm{a} 002$
19. Харари, Ф. Теория графов [Текст] / Ф. Харари; пер. с англ. - М.: 1973. -300 c.

20. Богданов, С. Н. Холодильная техника. Свойства веществ. Справочник [Текст] / С. Н. Богданов, О. П. Иванов, Ф. В. Куприянова. - М.: Агропромиздат, 1985. -208 c.

21. Томановская, В. Ф. Фреоны. Свойства и применение [Текст] / В. Ф. Томановская, Б. Е. Колотова. Л.: Химия, 1970. - 184 с.

22. Kudchadker, A. P. The Critical Constants of Organic Substances [Text] / A. P. Kudchadker, G. H. Alani, B. J. Zwolinski // Chem. Rev. - 1968. - Vol. 68, Issue 6. P. 659-735.

23. Филиппов, Л. П. Подобие свойств веществ [Текст] / Л. П. Филиппов. - М.: МГУ, 1978. - 256 с.

24. Кругляк, Ю. А. Новые инварианты взвешенных графов и их использование для расчета критических свойств фреонов [Текст] / Ю. А. Кругляк, И. В. Передунова. - Деп. в УкрНИИНТИ 5.07 .88 И767-Ук88, 1988. -65 с.

25. Передунова, И. В. Инварианты взвешенных графов в расчетах критических свойств алканов, фреонов и их $\mathrm{Si-аналогов} \mathrm{[Текст]:} \mathrm{дис.} \mathrm{…} \mathrm{к.т.н.:} \mathrm{05.13.16} \mathrm{/}$ И. В. Передунова. - Новосибирск, 1990. - 185 с. - Режим доступа: www.tekhnosfera.com/invarianty-vzveshennyhgrafov-v-raschetah-kriticheskih-svoystv-alkanov-freonov-i-ihst-analogov

\section{References}

1. Balaban, A. T. (1979). Chemical Graphs. XXXIV. Five New Topological Indices for the Branching of Tree-like Graphs. Theor. chim. Acta, 53 (4), 355-375.

2. Lovasz, L., Plammer, M. D. (1986). Matching Theory. Akademiai, Budapest, 653.

3. King, R. (Ed.) (1979). Chemical Applications of Topology and Graph Theory. N.Y.: Wiley, 605.

4. Perelstein, I. I., Parushin, U. B. (1984). Thermodynamical and thermophysical properties of refrigerants for Refrigerators and Heat Pumps. Moscow, 232.

5. Viktorov, M. M. (1977). Methods of Calculations of Physical-Chemical Properties and Applied Computations. Lviv: Chemistry, 360.

6. Read, R., Prausnitz, J., Sherwood, T. (1976). Properties of Gases and Liquids. N.Y.: Wiley, 605.

7. Filippov, L. P., Vorobeva, E. V., Okhotsimsky, A. D., Tolstunov, D. A. (1986) Razvitie metodov rascheta i prognozirovanija svojstv zhidkostej. Study of structure, heat movement and properties of liquids, Moskau: MSU, 141-176.

8. Morachevsky, A. G., Sladkov, I. B. (1987). Physicochemical properties of molecular inorganic substancies. Lviv: Chemistry, 192.

9. Jain, D. V. S., Singh, S., Gombar, V. (1984). Correlation Between Topological Features and Physicochemical Properties of Molecules. Proc. Indian Acad. Sci. (Chem. Sci.), 93 (6), 927-945.

10. Kruglyak, Yu. A., Peredunova, I. V. (1986). Establishing «structure - property» relationship for heteroconjugated molecules with the use of the weighted graph invariants and their informational analogues. VII All-union conference "Using computers in chemical research and molecular spectroscopy", Book of abstracts, Riga, 115-117.

11. Knop J. V., Trinajstic, N. (1980). Chemical Graph Theory. 11. On the Graph Theoretical Polynomials of Conjugated Structures. Intern. J. Quantum Chem., Quantum Chem. Symp, 14 (S14), 503-520. doi: 10.1002/qua.560180853

12. Godsil, C. D., Gutman, I. (1981). Some Remarks on the Matching Polynomial and Its Zeros. Croat. Chem. Acta, 54 (1), 53-59. 
13. Godsil, C. D. (1981). Matchings and Walks in
Graphs. J. Graph Theory, 5, 285-297. doi: $10.1002 /$ jgt.3190050310

14. Hosoya, H., Ohkami, N. J. (1983). Operator Technique for Obtaining the Recursion Formulae of Characteristic and Matching Polynomials as Applied to Polyhex Graphs. Comput. Chem, 4 (4), 585-593. doi: $10.1002 /$ jcc. 540040418

15. Hosoya, H. (1971). Topological Index. A Newly Proposed Quantity Characterizing the Topological Nature of Structural Isomers of Saturated Hydrocarbons. Bull. Chem. Soc. Japan, 44 (9), 2332-2339.

16. Farrel, E. J. (1979). An Introduction to Matching Polynomials. J. Combinatorial Theory, 27 (1), 75-86. doi: 10.1016/0095-8956(79)90070-4

17. Gutman, I., Shalabi, A., Naturforsch, Z. (1984). Topological Properties of Benzoid Systems. XXIX. On Hosoya's Topological Index, 39a, 797-799.

18. Gutman, I., Milun, M., Trinajstic, N. (1977). Graph Theory and Molecular Orbitals, 19. Nonparametric Resonance Energies of Arbitrary Cojugated Systems. J. Amer. Chem. Soc., 99 (6), 1692-1703. doi: 10.1021/ja00448a002
19. Harary, F. (1969). Graph Theory. London: Addison-Wesley, 310.

20. Bogdanov, S. N., Ivanov, O. P., Kupriyanova, F. V. (1985). Refrigerating Technology. Properties of Substances. Moscow: Agropromizdat, 208.

21. Tomanovskaya, V. F., Kolotova, B. E. (1970). Freons. Properties and Applications. Leningrad: Chemistry, 184.

22. Kudchadker, A. P., Alani, G. H., Zwolinski, B. J. (1968). The Critical Constants of Organic Substances. Chem. Rev., 68, 659-735.

23. Filippov, L. P. (1978). Similarity of Properties of Substances. Moscow.: MSU, 256.

24. Kruglyak Yu. A., Peredunova, I. V. (1988). New invariants of the weighted graphs and their use for calculation of critical properties of freons. Deposited to UkrNIINTI 5.07.88 I767-Uk88, 65.

25. Peredunova, I. V. (1990). Invariants of the weighted graphs for calculation of critical properties of alkanes, freons and their Si-analogues. Novosibirstk, 185. Available at: www.tekhnosfera.com/invarianty-vzveshennyhgrafov-v-raschetah-kriticheskih-svoystv-alkanov-freonov-i-ihst-analogov

Рекомендовано до публікації д-р фіз.-мат. наук Глушков О. В. Дата надходження рукопису 30.09.2014

Кругляк Юрий Алексеевич, доктор химических наук, профессор, кафедра информационных технологий, Одесский государственный экологический университет, ул. Львовская, 15, г. Одесса, 65016, Украина

E-mail: quantumnet@yandex.ua

Передунова Ирина Викторовна, кандидат технических наук, доцент, кафедра информационных технологий, Одесский государственный экологический университет, ул. Львовская, 15, г. Одесса, 65016, Украина

E-mail: kaf-infotech@odeku.edu.ua, 